J. N. Alonso Álvarez • J. M. Fernández Vilaboa •

R. González Rodríguez • C. Soneira Calvo

\title{
Yetter-Drinfeld categories associated with a weak braided Hopf algebra
}

Received: 16 April 2012 / Accepted: 27 July 2012 / Published online: 13 September 2012

(C) The Author(s) 2012. This article is published with open access at Springerlink.com

\begin{abstract}
In a previous paper, the authors introduced the monoidal category of left-left Yetter-Drinfeld modules over a weak braided Hopf algebra in a strict monoidal category. The main goal of this work is to define the categories of right-right, left-right and right-left Yetter-Drinfeld modules over a weak braided Hopf algebra and prove that there exists a categorical equivalence between all of them. We also establish the categorical equivalences by changing the weak braided Hopf algebra D by its (co)opposite. Finally, the general results are illustrated with an example coming from the projections of weak braided Hopf algebras.
\end{abstract}

Mathematics Subject Classification $16 \mathrm{~W} 30 \cdot 18 \mathrm{D} 10 \cdot 16 \mathrm{~T} 05 \cdot 16 \mathrm{~T} 25 \cdot 81 \mathrm{R} 50$

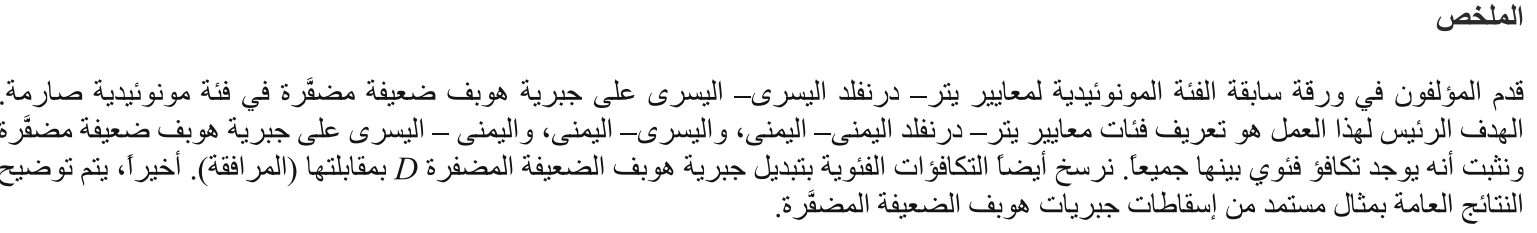

\section{Introduction}

To study the projections of Hopf algebras, Radford [13] establishes conditions that led to the notion of Yetter-Drinfeld module introduced by Yetter [17] in order to explain the relationship between different theories in mathematics and physics, as low dimensional topology, knots and links, Hopf algebras, quantum integrable systems, and exactly solvable models in statistical mechanics. In this sense, every Yetter-Drinfeld module gives rise to a solution of the quantum Yang-Baxter equation, as was proved in [9], and if $H$ is a finite Hopf algebra

J. N. Alonso Álvarez (凶)

Departamento de Matemáticas, Universidade de Vigo, Campus Universitario Lagoas-Marcosende, 36280 Vigo, Spain

E-mail: jnalonso@uvigo.es

J. M. Fernández Vilaboa

Departamento de Álxebra, Universidade de Santiago de Compostela, 15771 Santiago de Compostela, Spain

E-mail: josemanuel.fernandez@usc.es

R. González Rodríguez

Departamento de Matemática Aplicada II, Universidade de Vigo, Campus Universitario Lagoas-Marcosende, 36310 Vigo, Spain

E-mail: rgon@dma.uvigo.es

C. Soneira Calvo

Departamento de Pedagoxía e Didáctica, Universidade da Coruña, Campus Universitario de Elviña, 15007 A Coruña, Spain

E-mail: carlos.soneira@udc.es 
in a symmetric monoidal category $\mathcal{C}$, the category ${ }_{H}^{H} \mathcal{Y D}$ of left-left Yetter-Drinfeld modules is isomorphic to the category of modules over the quantum double, which was originally conceived to find solutions of the Yang-Baxter equation via universal matrices. Continuing with physical applications, in a symmetric category the notion of projection is a generalization of that of bosonization introduced by Majid [11] and allows to give, for $\mathrm{H}$ a quasitriangular Hopf algebra, an interpretation of crossed products in terms of quantum algebras of observables of dynamical systems, as well as in quantum group gauge theory. Just to define the quantum double it must be considered the opposite Hopf algebra $H^{\mathrm{op}}$. Moreover, as was proved in [14], depending on the smash product we use to construct the quantum double, the category of modules over it is isomorphic to the category ${ }_{H} \mathcal{Y} \mathcal{D}^{H}$ of left-right Yetter-Drinfeld modules. These phenomena lead to take into account the various (co)algebra structures obtained when modifying successively the (co)multiplication by a twist, as well as the various Yetter-Drinfeld categories emerging when changing the side of the module and/or comodule structure, namely ${ }_{H}^{H} \mathcal{Y D}, \mathcal{Y} \mathcal{D}_{H}^{H},{ }_{H} \mathcal{Y} \mathcal{D}^{H}$ and ${ }^{H} \mathcal{Y} \mathcal{D}_{H}$. In turn, it brings the notice to the study of the relations between all these categories. The milestone in the categorical treatment of Yetter-Drinfeld modules appears in [15], where the various categories of Yetter-Drinfeld modules are defined over a Hopf algebra in a symmetric category and proved to be categorically equivalent. Actually, in this classic case, the symmetry of the category is explicitly used to define all the notions of Yetter-Drinfeld modules.

On the other hand, weak Hopf algebras (or quantum groupoids in the terminology of Nikshych and Vainerman [12]) were introduced by Böhm, Nill and Szlachányi [5] as a generalization of Hopf algebras and groupoid algebras. The main difference with other Hopf algebraic constructions, such as quasi-Hopf algebras and rational Hopf algebras, is that weak Hopf algebras are coassociative but the coproduct is not required to preserve the unit, or equivalently, the counit is not an algebra morphism. There are good motivations to studying weak Hopf algebras. Group algebras and their duals are the natural examples of Hopf algebras, groupoid algebras and their duals provide examples of weak Hopf algebras, and secondly, these algebraic structures have a remarkable connection with the theory of algebra extensions, important applications in the study of dynamical twists of Hopf algebras and a deep link with quantum field theories and operator algebras [12].

In [1], the authors introduce the notions of weak Yang-Baxter operator and weak braided Hopf algebra. Roughly speaking, a weak braided Hopf algebra in a strict monoidal category is an algebra-coalgebra with a weak Yang-Baxter operator, satisfying some compatibility conditions. This definition generalizes the one introduced by Takeuchi [16], i.e., the definition of braided Hopf algebra, and the classical notions of Hopf algebra and Hopf algebra in a braided category. Moreover, as particular instances, the definition of weak Hopf algebra is recovered and, if the weak Yang-Baxter operator is the braiding of a braided category, the notion of weak Hopf algebra in a braided monoidal setting is formulated. The first non-trivial example of weak braided Hopf algebra can be obtained by modifying the algebraic structure of a Hopf algebra $D$ in the non-strict braided monoidal category ${ }_{H}^{H} \mathcal{Y D}$. In [4], the authors introduce the notion of weak operator and use it to establish a left-left Yetter-Drinfeld module theory in a general strict monoidal category.

The present work continues that study. Our main motivation is to extend the results of [15] to the general case, dealing with a weak braided Hopf algebra in a monoidal ambient category $\mathcal{C}$ that is not assumed to be equipped with a braiding. More precisely, we define the categories of left-left, right-right, left-right and right-left Yetter-Drinfeld modules over a weak braided Hopf algebra $D$, generalizing the ones introduced in [15] for a symmetric category. Moreover, if $\mathcal{C}$ is a braided category with braiding $c$, taking the suitable weak operator we obtain as particular instances the definitions of Yetter-Drinfeld modules for this setting. On the other hand, by changing the weak braided Hopf algebra $D$ by its (co)opposite we also introduce the various Yetter-Drinfeld categories over $D^{\mathrm{op}}, D^{\text {coop }}, D^{\text {op }}{ }^{\text {coop }}$ and $D^{\text {coop }}$. We show that all of these categories are equivalent. Finally, we apply our results to an example coming from projections of weak braided Hopf algebras [3]. The organization of the paper is the following. After the introduction, in Sect. 2, the general framework is stated recalling the definitions of weak Yang-Baxter operator, weak braided bialgebra and weak braided Hopf algebra; as well as that of weak operator and its main properties, including the notion of compatibility for the (co)module structures that emerges naturally when dealing with the Yetter-Drinfeld categories. In Sect. 3, we introduce the definitions of the various Yetter-Drinfeld module categories over an arbitrary weak braided Hopf algebra, we prove that all of them are categorically equivalent and provide an example of application.

\section{Weak operators}

In this paper, we denote a monoidal category $\mathcal{C}$ as $(\mathcal{C}, \otimes, K, \mathfrak{a}, \mathfrak{l}, \mathfrak{r})$ where $\mathcal{C}$ is a category and $\otimes$ (tensor product) provides $\mathcal{C}$ with a monoidal structure with unit object $K$ whose associative constraint is denoted by $\mathfrak{a}$ and whose left and right unit constraints are given by $\mathfrak{l}$ and $\mathfrak{r}$, respectively (see [10]). 
We denote the class of objects of $\mathcal{C}$ by $|\mathcal{C}|$ and for each object $M \in|\mathcal{C}|$, the identity morphism by $i d_{M}: M \rightarrow M$. For simplicity of notation, given objects $M, N, P$ in $\mathcal{C}$ and a morphism $f: M \rightarrow N$, we write $P \otimes f$ for $i d_{P} \otimes f$ and $f \otimes P$ for $f \otimes i d_{P}$.

From now on we assume that $\mathcal{C}$ is strict and that it has split idempotents, i.e., for every morphism $\nabla_{Y}: Y \rightarrow$ $Y$ such that $\nabla_{Y}=\nabla_{Y} \circ \nabla_{Y}$ there exist an object $Z$ (called the image of $\nabla_{Y}$ ) and morphisms $i_{Y}: Z \rightarrow Y$ and $p_{Y}: Y \rightarrow Z$ such that $\nabla_{Y}=i_{Y} \circ p_{Y}$ and $p_{Y} \circ i_{Y}=i d_{Z}$. There is not loss of generality in assuming the strict character for $\mathcal{C}$ because it is well known that given a monoidal category we can construct a strict monoidal category $\mathcal{C}^{s t}$ which is tensor equivalent to $\mathcal{C}$ (see [8] for the details); neither in assuming that $\mathcal{C}$ admits split idempotents, taking into account that for a given category $\mathcal{C}$ there exists an universal embedding $\mathcal{C} \rightarrow \hat{\mathcal{C}}$ such that $\hat{\mathcal{C}}$ admits split idempotents, as was proved in [8]. A braided monoidal category $\mathcal{C}$ is a monoidal category in which there is for all $M$ and $N$ in $\mathcal{C}$ a natural isomorphism $c_{M, N}: M \otimes N \rightarrow N \otimes M$, called the braiding, satisfying the Hexagon Axiom (see [7] for generalities). If the braiding satisfies $c_{N, M} \circ c_{M, N}=i d_{M \otimes N}$ for all $M, N$ in $\mathcal{C}$, the category is called symmetric.

Definition 2.1 An algebra in $\mathcal{C}$ is a triple $A=\left(A, \eta_{A}, \mu_{A}\right)$ where $A$ is an object in $\mathcal{C}$ and $\eta_{A}: K \rightarrow A$ (unit), $\mu_{A}: A \otimes A \rightarrow A$ (product) are morphisms in $\mathcal{C}$ such that $\mu_{A} \circ\left(A \otimes \eta_{A}\right)=i d_{A}=\mu_{A} \circ\left(\eta_{A} \otimes A\right)$, $\mu_{A} \circ\left(A \otimes \mu_{A}\right)=\mu_{A} \circ\left(\mu_{A} \otimes A\right)$. Given two algebras $A=\left(A, \eta_{A}, \mu_{A}\right)$ and $B=\left(B, \eta_{B}, \mu_{B}\right), f: A \rightarrow B$ is an algebra morphism if $f \circ \eta_{A}=\eta_{B}, \mu_{B} \circ(f \otimes f)=f \circ \mu_{A}$.

A coalgebra in $\mathcal{C}$ is a triple $D=\left(D, \varepsilon_{D}, \delta_{D}\right)$ where $D$ is an object in $\mathcal{C}$ and $\varepsilon_{D}: D \rightarrow K$ (counit), $\delta_{D}: D \rightarrow D \otimes D$ (coproduct) are morphisms in $\mathcal{C}$ such that $\left(\varepsilon_{D} \otimes D\right) \circ \delta_{D}=i d_{D}=\left(D \otimes \varepsilon_{D}\right) \circ \delta_{D}$, $\left(\delta_{D} \otimes D\right) \circ \delta_{D}=\left(D \otimes \delta_{D}\right) \circ \delta_{D}$. If $D=\left(D, \varepsilon_{D}, \delta_{D}\right)$ and $E=\left(E, \varepsilon_{E}, \delta_{E}\right)$ are coalgebras, $f: D \rightarrow E$ is a coalgebra morphism if $\varepsilon_{E} \circ f=\varepsilon_{D},(f \otimes f) \circ \delta_{D}=\delta_{E} \circ f$.

If $A$ is an algebra, $B$ is a coalgebra and $\alpha: B \rightarrow A, \beta: B \rightarrow A$ are morphisms, we define the convolution product by $\alpha * \beta=\mu_{A} \circ(\alpha \otimes \beta) \circ \delta_{B}$.

If $\left(D, \eta_{D}, \mu_{D}\right)$ is an algebra in $\mathcal{C}$, the pair $\left(M, \varphi_{M}\right)$, with $M \in|\mathcal{C}|$ and $\varphi_{M}: D \otimes M \rightarrow D$ is said to be a left $D$-module if $\varphi_{M} \circ\left(\eta_{D} \otimes M\right)=i d_{M}$ and $\varphi_{M} \circ\left(D \otimes \varphi_{M}\right)=\varphi_{M} \circ\left(\mu_{D} \otimes M\right)$. Given two left $D$-modules $\left(M, \varphi_{M}\right)$ and $\left(N, \varphi_{N}\right), f: M \rightarrow N$ is a morphism of left $D$-modules if $\varphi_{N} \circ(D \otimes f)=f \circ \varphi_{M}$.

If $\left(D, \varepsilon_{D}, \delta_{D}\right)$ is a coalgebra in $\mathcal{C}$, the pair $\left(M, \varrho_{M}\right)$ with $M \in|\mathcal{C}|$ and $\varrho_{M}: M \rightarrow D \otimes M$ is said to be a left $D$-comodule if $\left(\varepsilon_{D} \otimes M\right) \circ \varrho_{M}=i d_{M}$ and $\left(D \otimes \varrho_{M}\right) \circ \varrho_{M}=\left(\delta_{D} \otimes M\right) \circ \varrho_{M}$. Given two left $D$-comodules $\left(M, \varrho_{M}\right)$ and $\left(N, \varrho_{N}\right), f: M \rightarrow N$ is a morphism of left $D$-comodules if $\varrho_{N} \circ f=(D \otimes f) \circ \varrho_{M}$. The notions of right $D$-(co)module are defined analogously.

Definition 2.2 Let $D$ be in $\mathcal{C}$ and let $t_{D, D}: D \otimes D \rightarrow D \otimes D$ be a morphism in $\mathcal{C}$. We will say that $t_{D, D}$ satisfies the Yang-Baxter equation if

$$
\left(t_{D, D} \otimes D\right) \circ\left(D \otimes t_{D, D}\right) \circ\left(t_{D, D} \otimes D\right)=\left(D \otimes t_{D, D}\right) \circ\left(t_{D, D} \otimes D\right) \circ\left(D \otimes t_{D, D}\right) .
$$

Weak Yang-Baxter operators are generalizations of Yang-Baxter operators (see [7]) and were introduced by Alonso et al. [1]. Roughly speaking, a weak Yang-Baxter operator is an endomorphism satisfying the YangBaxter equation that restricted to the image of a suitable idempotent is an isomorphism. In [3] we prove that one axiom of the original definition can be dropped and in [4] we give a list of examples. We rewrite the improved definition:

Definition 2.3 Let $D$ be in $\mathcal{C}$. A weak Yang-Baxter operator is a morphism $t_{D, D}: D \otimes D \rightarrow D \otimes D$ in $\mathcal{C}$ such that:

(a1) $t_{D, D}$ satisfies the Yang-Baxter equation.

(a2) There exists an idempotent morphism $\nabla_{D, D}: D \otimes D \rightarrow D \otimes D$ satisfying the following identities:

$(\mathrm{a} 2-1)\left(\nabla_{D, D} \otimes D\right) \circ\left(D \otimes \nabla_{D, D}\right)=\left(D \otimes \nabla_{D, D}\right) \circ\left(\nabla_{D, D} \otimes D\right)$,

$(\mathrm{a} 2-2)\left(\nabla_{D, D} \otimes D\right) \circ\left(D \otimes t_{D, D}\right)=\left(D \otimes t_{D, D}\right) \circ\left(\nabla_{D, D} \otimes D\right)$,

$(\mathrm{a} 2-3)\left(t_{D, D} \otimes D\right) \circ\left(D \otimes \nabla_{D, D}\right)=\left(D \otimes \nabla_{D, D}\right) \circ\left(t_{D, D} \otimes D\right)$,

(a2-4) $t_{D, D} \circ \nabla_{D, D}=\nabla_{D, D} \circ t_{D, D}=t_{D, D}$.

(a3) There exists a morphism $t_{D, D}^{\prime}: D \otimes D \rightarrow D \otimes D$ such that:

(a3-1) The morphism $p_{D, D} \circ t_{D, D} \circ i_{D, D}: D \times D \rightarrow D \times D$ is an isomorphism with inverse $p_{D, D} \circ t_{D, D}^{\prime} \circ i_{D, D}$ : $D \times D \rightarrow D \times D$, where $p_{D, D}$ and $i_{D, D}$ are the morphisms such that $i_{D, D} \circ p_{D, D}=\nabla_{D, D}$ and $p_{D, D} \circ i_{D, D}=i d_{D \times D}$ and also $D \times D$ is the image of $\nabla_{D, D}$.

(a3-2) $t_{D, D}^{\prime} \circ \nabla_{D, D}=\nabla_{D, D} \circ t_{D, D}^{\prime}=t_{D, D}^{\prime}$. 
Note that if $\nabla_{D, D}=i d_{D \otimes D}$ then $t_{D, D}$ is an isomorphism and we recover the definition of Yang-Baxter operator introduced by Joyal and Street in [7]. Also, by [[1], Definition 1.2 and Proposition 1.3], we get that $t_{D, D}$ is a weak Yang-Baxter operator with associated idempotent $\nabla_{D, D}$ if and only if so is $t_{D, D}^{\prime}$. Moreover, in this case

$$
t^{\prime}{ }_{D, D} \circ t_{D, D}=t_{D, D} \circ t^{\prime}{ }_{D, D}=\nabla_{D, D}
$$

Finally, using the identities (2)-(5) of [1] we obtain:

$$
\begin{aligned}
& \left(D \otimes t_{D, D}\right) \circ\left(t_{D, D} \otimes D\right) \circ\left(D \otimes t_{D, D}^{\prime}\right)=\left(t_{D, D}^{\prime} \otimes D\right) \circ\left(D \otimes t_{D, D}\right) \circ\left(t_{D, D} \otimes D\right), \\
& \left(t_{D, D} \otimes D\right) \circ\left(D \otimes t_{D, D}\right) \circ\left(t_{D, D}^{\prime} \otimes D\right)=\left(D \otimes t_{D, D}^{\prime}\right) \circ\left(t_{D, D} \otimes D\right) \circ\left(D \otimes t_{D, D}\right), \\
& \left(D \otimes t_{D, D}^{\prime}\right) \circ\left(t_{D, D}^{\prime} \otimes D\right) \circ\left(D \otimes t_{D, D}\right)=\left(t_{D, D} \otimes D\right) \circ\left(D \otimes t_{D, D}^{\prime}\right) \circ\left(t_{D, D}^{\prime} \otimes D\right), \\
& \left(t_{D, D}^{\prime} \otimes D\right) \circ\left(D \otimes t_{D, D}^{\prime}\right) \circ\left(t_{D, D} \otimes D\right)=\left(D \otimes t_{D, D}\right) \circ\left(t_{D, D}^{\prime} \otimes D\right) \circ\left(D \otimes t_{D, D}^{\prime}\right) .
\end{aligned}
$$

Now we recall the definition of weak braided bialgebra and weak braided Hopf algebra introduced by Alonso et al. [1]. The interested reader can see the main properties in Sect. 2 of [2].

Definition 2.4 A weak braided bialgebra (WBB for short) $D$ is an object in $\mathcal{C}$ with an algebra structure $\left(D, \eta_{D}, \mu_{D}\right)$ and a coalgebra structure $\left(D, \varepsilon_{D}, \delta_{D}\right)$ such that there exists a weak Yang-Baxter operator $t_{D, D}$ : $D \otimes D \rightarrow D \otimes D$ with associated idempotent $\nabla_{D, D}$ satisfying the following conditions:

(b1-1) $\mu_{D} \circ \nabla_{D, D}=\mu_{D}$,

(b1-2) $\nabla_{D, D} \circ\left(\mu_{D} \otimes D\right)=\left(\mu_{D} \otimes D\right) \circ\left(D \otimes \nabla_{D, D}\right)$,

(b1-3) $\nabla_{D, D} \circ\left(D \otimes \mu_{D}\right)=\left(D \otimes \mu_{D}\right) \circ\left(\nabla_{D, D} \otimes D\right)$.

(b2-1) $\nabla_{D, D} \circ \delta_{D}=\delta_{D}$,

$(\mathrm{b} 2-2)\left(\delta_{D} \otimes D\right) \circ \nabla_{D, D}=\left(D \otimes \nabla_{D, D}\right) \circ\left(\delta_{D} \otimes D\right)$,

(b2-3) $\left(D \otimes \delta_{D}\right) \circ \nabla_{D, D}=\left(\nabla_{D, D} \otimes D\right) \circ\left(D \otimes \delta_{D}\right)$.

(b3) The morphisms $\mu_{D}$ and $\delta_{D}$ commute with $t_{D, D}$, i.e.,

(b3-1) $t_{D, D} \circ\left(\mu_{D} \otimes D\right)=\left(D \otimes \mu_{D}\right) \circ\left(t_{D, D} \otimes D\right) \circ\left(D \otimes t_{D, D}\right)$,

(b3-2) $t_{D, D} \circ\left(D \otimes \mu_{D}\right)=\left(\mu_{D} \otimes D\right) \circ\left(D \otimes t_{D, D}\right) \circ\left(t_{D, D} \otimes D\right)$,

(b3-3) $\left(\delta_{D} \otimes D\right) \circ t_{D, D}=\left(D \otimes t_{D, D}\right) \circ\left(t_{D, D} \otimes D\right) \circ\left(D \otimes \delta_{D}\right)$,

(b3-4) $\left(D \otimes \delta_{D}\right) \circ t_{D, D}=\left(t_{D, D} \otimes D\right) \circ\left(D \otimes t_{D, D}\right) \circ\left(\delta_{D} \otimes D\right)$.

(b4) $\delta_{D} \circ \mu_{D}=\left(\mu_{D} \otimes \mu_{D}\right) \circ\left(D \otimes t_{D, D} \otimes D\right) \circ\left(\delta_{D} \otimes \delta_{D}\right)$.

(b5) $\varepsilon_{D} \circ \mu_{D} \circ\left(\mu_{D} \otimes D\right)=\left(\left(\varepsilon_{D} \circ \mu_{D}\right) \otimes\left(\varepsilon_{D} \circ \mu_{D}\right)\right) \circ\left(D \otimes \delta_{D} \otimes D\right)$

$=\left(\left(\varepsilon_{D} \circ \mu_{D}\right) \otimes\left(\varepsilon_{D} \circ \mu_{D}\right)\right) \circ\left(D \otimes\left(t_{D, D}^{\prime} \circ \delta_{D}\right) \otimes D\right)$.

(b6) $\left(\delta_{D} \otimes D\right) \circ \delta_{D} \circ \eta_{D}=\left(D \otimes \mu_{D} \otimes D\right) \circ\left(\left(\delta_{D} \circ \eta_{D}\right) \otimes\left(\delta_{D} \circ \eta_{D}\right)\right)$ $=\left(D \otimes\left(\mu_{D} \circ t_{D, D}^{\prime}\right) \otimes D\right) \circ\left(\left(\delta_{D} \circ \eta_{D}\right) \otimes\left(\delta_{D} \circ \eta_{D}\right)\right)$.

A weak braided bialgebra $D$ is said to be a weak braided Hopf algebra (WBHA for short) if:

(b7) There exists a morphism $\lambda_{D}: D \rightarrow D$ in $\mathcal{C}$ (called the antipode of $D$ ) satisfying:

$(\mathrm{b} 7-1) i d_{D} * \lambda_{D}=\left(\left(\varepsilon_{D} \circ \mu_{D}\right) \otimes D\right) \circ\left(D \otimes t_{D, D}\right) \circ\left(\left(\delta_{D} \circ \eta_{D}\right) \otimes D\right)$,

$(\mathrm{b} 7-2) \lambda_{D} * i d_{D}=\left(D \otimes\left(\varepsilon_{D} \circ \mu_{D}\right)\right) \circ\left(t_{D, D} \otimes D\right) \circ\left(D \otimes\left(\delta_{D} \circ \eta_{D}\right)\right)$,

(b7-3) $\lambda_{D} * i d_{D} * \lambda_{D}=\lambda_{D}$.

Note that the antipode is unique, antimultiplicative, anticomultiplicative and leaves the unit and counit invariant (see [2]).

Let $D, B$ be WBHA. We will say that $f: D \rightarrow B$ is a morphism of WBHA if $f$ is an algebra coalgebra morphism and $t_{B, B} \circ(f \otimes f)=(f \otimes f) \circ t_{D, D}$ and $t_{B, B}^{\prime} \circ(f \otimes f)=(f \otimes f) \circ t_{D, D}^{\prime}$. It is not difficult to see that, if $f: D \rightarrow B$ is a morphism of weak braided Hopf algebras, then $f \circ \lambda_{D}=\lambda_{B} \circ f$ (see [1] for details). Moreover, by (2) we obtain $\nabla_{B, B} \circ(f \otimes f)=(f \otimes f) \circ \nabla_{D, D}$.

Note that, if $\mathcal{C}$ is symmetric and $t_{D, D}=t_{D, D}^{\prime}$ is the braiding of the category, then if $D$ is a WBHA, $\nabla_{D, D}=i d_{D \otimes D}$ and we obtain the well known definition of weak Hopf algebra. When $\mathcal{C}$ is a braided category with braiding $c$ and $t_{D, D}=c_{D, D}$ and $t_{D, D}^{\prime}=c_{D, D}^{-1}$, we have that $\nabla_{D, D}=i d_{D \otimes D}$ and $D$ is called a weak Hopf algebra in $\mathcal{C}$. Also, braided Hopf algebras, in the sense of Takeuchi [16], are WBHA. Finally, we can obtain more examples if we consider Hopf algebras in the non strict braided monoidal category of left-left 
Yetter-Drinfeld modules over a weak Hopf algebra $H$ which lives in a strict monoidal category $\mathcal{C}$ with split idempotents (see Examples 1.6. of [4]).

2.5 The morphisms $\Pi_{D}^{L}, \Pi_{D}^{R}, \bar{\Pi}_{D}^{L}$ and $\bar{\Pi}_{D}^{R}$ are defined as:

$$
\begin{aligned}
& \Pi_{D}^{L}=\left(\left(\varepsilon_{D} \circ \mu_{D}\right) \otimes D\right) \circ\left(t_{D, D} \otimes D\right) \circ\left(\left(\delta_{D} \circ \eta_{D}\right) \otimes D\right), \\
& \Pi_{D}^{R}=\left(D \otimes\left(\varepsilon_{D} \circ \mu_{D}\right)\right) \circ\left(D \otimes t_{D, D}\right) \circ\left(\left(D \otimes\left(\delta_{D} \circ \eta_{D}\right)\right),\right. \\
& \bar{\Pi}_{D}^{L}=\left(D \otimes\left(\varepsilon_{D} \circ \mu_{D}\right)\right) \circ\left(\left(\delta_{D} \circ \eta_{D}\right) \otimes D\right), \\
& \bar{\Pi}_{D}^{R}=\left(\left(\varepsilon_{D} \circ \mu_{D}\right) \otimes D\right) \circ\left(D \otimes\left(\delta_{D} \circ \eta_{D}\right)\right) .
\end{aligned}
$$

It is easy to see that they are idempotent and leave the unit and the counit invariant. Moreover, they satisfy the following equalities:

$$
\Pi_{D}^{L}=i d_{D} * \lambda_{D}, \quad \Pi_{D}^{R}=\lambda_{D} * i d_{D}, \quad \lambda_{D}=\lambda_{D} * \Pi_{D}^{L}=\Pi_{D}^{R} * \lambda_{D} .
$$

Concerning to the behavior of the antipode, we know that

$$
t_{D, D} \circ\left(\lambda_{D} \otimes D\right)=\left(D \otimes \lambda_{D}\right) \circ t_{D, D}, \quad t_{D, D}^{\prime} \circ\left(D \otimes \lambda_{D}\right)=\left(\lambda_{D} \otimes D\right) \circ t_{D, D}^{\prime}
$$

and

$$
t_{D, D} \circ\left(D \otimes \lambda_{D}\right)=\left(\lambda_{D} \otimes D\right) \circ t_{D, D}, \quad t_{D, D}^{\prime} \circ\left(\lambda_{D} \otimes D\right)=\left(D \otimes \lambda_{D}\right) \circ t_{D, D}^{\prime}
$$

(See Proposition 2.12 of [2] for details).

Now we introduce the definition of weak operator which generalizes the notion of weak Yang-Baxter operator.

Definition 2.6 Let $D$ be a WBHA and let $M$ be an object of $\mathcal{C}$. A weak operator between $M$ and $D$, (from now on referred as $(M, D)$-WO) is defined as a quadruple $\left(r_{M}, r_{M}^{\prime}, s_{M}, s_{M}^{\prime}\right)$ comprised of four morphisms in $\mathcal{C}$ :

$$
\begin{array}{ll}
r_{M}: M \otimes D \rightarrow D \otimes M, & r_{M}^{\prime}: D \otimes M \rightarrow M \otimes D, \\
s_{M}: D \otimes M \rightarrow M \otimes D, & s_{M}^{\prime}: M \otimes D \rightarrow D \otimes M,
\end{array}
$$

satisfying the following conditions:

(c1)

$(\mathrm{c} 1-1)\left(D \otimes r_{M}\right) \circ\left(r_{M} \otimes D\right) \circ\left(M \otimes t_{D, D}\right)=\left(t_{D, D} \otimes M\right) \circ\left(D \otimes r_{M}\right) \circ\left(r_{M} \otimes D\right)$,

$(\mathrm{c} 1-2)\left(r_{M}^{\prime} \otimes D\right) \circ\left(D \otimes r_{M}^{\prime}\right) \circ\left(t_{D, D} \otimes M\right)=\left(M \otimes t_{D, D}\right) \circ\left(r_{M}^{\prime} \otimes D\right) \circ\left(D \otimes r_{M}^{\prime}\right)$,

$(\mathrm{c} 1-3)\left(s_{M} \otimes D\right) \circ\left(D \otimes s_{M}\right) \circ\left(t_{D, D} \otimes M\right)=\left(M \otimes t_{D, D}\right) \circ\left(s_{M} \otimes D\right) \circ\left(D \otimes s_{M}\right)$,

$(\mathrm{c} 1-4)\left(D \otimes s_{M}^{\prime}\right) \circ\left(s_{M}^{\prime} \otimes D\right) \circ\left(M \otimes t_{D, D}\right)=\left(t_{D, D} \otimes M\right) \circ\left(D \otimes s_{M}^{\prime}\right) \circ\left(s_{M}^{\prime} \otimes D\right)$. The analogous equalities with $t_{D, D}^{\prime}$ instead of $t_{D, D}$ are also required to be satisfied.

$(\mathrm{c} 2-1)\left(r_{M}^{\prime} \otimes D\right) \circ\left(D \otimes s_{M}\right) \circ\left(t_{D, D} \otimes M\right)=\left(M \otimes t_{D, D}\right) \circ\left(s_{M} \otimes D\right) \circ\left(D \otimes r_{M}^{\prime}\right)$,

$(\mathrm{c} 2-2)\left(s_{M} \otimes D\right) \circ\left(D \otimes r_{M}^{\prime}\right) \circ\left(t_{D, D}^{\prime} \otimes M\right)=\left(M \otimes t_{D, D}^{\prime}\right) \circ\left(r_{M}^{\prime} \otimes D\right) \circ\left(D \otimes s_{M}\right)$,

$(\mathrm{c} 2-3)\left(D \otimes s_{M}^{\prime}\right) \circ\left(r_{M} \otimes D\right) \circ\left(M \otimes t_{D, D}\right)=\left(t_{D, D} \otimes M\right) \circ\left(D \otimes r_{M}\right) \circ\left(s_{M}^{\prime} \otimes D\right)$,

$(\mathrm{c} 2-4)\left(D \otimes r_{M}\right) \circ\left(s_{M}^{\prime} \otimes D\right) \circ\left(M \otimes t_{D, D}^{\prime}\right)=\left(t_{D, D}^{\prime} \otimes M\right) \circ\left(D \otimes s_{M}^{\prime}\right) \circ\left(r_{M} \otimes D\right)$.

We want to point out that in this case, as in general for all the mixed equations along the paper, we cannot replace $t_{D, D}$ by $t_{D, D}^{\prime}$ or $t_{D, D}^{\prime}$ by $t_{D, D}$.

(c3) The morphisms: $\nabla_{r_{M}}:=r_{M}^{\prime} \circ r_{M}, \nabla_{r_{M}^{\prime}}:=r_{M} \circ r_{M}^{\prime}, \nabla_{s_{M}}:=s_{M}^{\prime} \circ s_{M}$ and $\nabla_{s_{M}^{\prime}}:=s_{M} \circ s_{M}^{\prime}$ satisfy

(c3-1) $\nabla_{r_{M}}=\left(\left(\left(\varepsilon_{D} \otimes M\right) \circ r_{M}\right) \otimes D\right) \circ\left(M \otimes \delta_{D}\right)=\left(M \otimes \mu_{D}\right) \circ\left(\left(\left(r_{M}^{\prime} \circ\left(\eta_{D} \otimes M\right)\right) \otimes D\right)\right.$,

(c3-2) $\nabla_{r_{M}^{\prime}}=\left(D \otimes\left(\left(M \otimes \varepsilon_{D}\right) \circ r_{M}^{\prime}\right)\right) \circ\left(\delta_{D} \otimes M\right)=\left(\mu_{D} \otimes M\right) \circ\left(D \otimes\left(r_{M} \circ\left(M \otimes \eta_{D}\right)\right)\right)$,

(c3-3) $\nabla_{s_{M}}=\left(D \otimes\left(\left(M \otimes \varepsilon_{D}\right) \circ s_{M}\right)\right) \circ\left(\delta_{D} \otimes M\right)=\left(\mu_{D} \otimes M\right) \circ\left(D \otimes\left(s_{M}^{\prime} \circ\left(M \otimes \eta_{D}\right)\right)\right)$,

(c3-4) $\nabla_{s_{M}^{\prime}}=\left(\left(\left(\varepsilon_{D} \otimes M\right) \circ s_{M}^{\prime}\right) \otimes D\right) \circ\left(M \otimes \delta_{D}\right)=\left(M \otimes \mu_{D}\right) \circ\left(\left(\left(s_{M} \circ\left(\eta_{D} \otimes M\right)\right) \otimes D\right)\right.$.

(c4-1) $r_{M} \circ\left(M \otimes \mu_{D}\right)=\left(\mu_{D} \otimes M\right) \circ\left(D \otimes r_{M}\right) \circ\left(r_{M} \otimes D\right)$,

$(\mathrm{c} 4-2) r_{M}^{\prime} \circ\left(\mu_{D} \otimes M\right)=\left(M \otimes \mu_{D}\right) \circ\left(r_{M}^{\prime} \otimes D\right) \circ\left(D \otimes r_{M}^{\prime}\right)$, 
(c4-3) $\left(D \otimes r_{M}\right) \circ\left(r_{M} \otimes D\right) \circ\left(M \otimes \delta_{D}\right)=\left(\delta_{D} \otimes M\right) \circ r_{M}$,

$(\mathrm{c} 4-4)\left(r_{M}^{\prime} \otimes D\right) \circ\left(D \otimes r_{M}^{\prime}\right) \circ\left(\delta_{D} \otimes M\right)=\left(M \otimes \delta_{D}\right) \circ r_{M}^{\prime}$,

(c4-5) $s_{M} \circ\left(\mu_{D} \otimes M\right)=\left(M \otimes \mu_{D}\right) \circ\left(s_{M} \otimes D\right) \circ\left(D \otimes s_{M}\right)$,

(c4-6) $s_{M}^{\prime} \circ\left(M \otimes \mu_{D}\right)=\left(\mu_{D} \otimes M\right) \circ\left(D \otimes s_{M}^{\prime}\right) \circ\left(s_{M}^{\prime} \otimes D\right)$,

$(\mathrm{c} 4-7)\left(s_{M} \otimes D\right) \circ\left(D \otimes s_{M}\right) \circ\left(\delta_{D} \otimes M\right)=\left(M \otimes \delta_{D}\right) \circ s_{M}$,

$(\mathrm{c} 4-8)\left(D \otimes s_{M}^{\prime}\right) \circ\left(s_{M}^{\prime} \otimes D\right) \circ\left(M \otimes \delta_{D}\right)=\left(\delta_{D} \otimes M\right) \circ s_{M}^{\prime}$.

$(\mathrm{c5-1})\left(M \otimes \lambda_{D}\right) \circ \nabla_{r_{M}}=\nabla_{r_{M}} \circ\left(M \otimes \lambda_{D}\right)$,

(c5-2) $\left(\lambda_{D} \otimes M\right) \circ \nabla_{r_{M}^{\prime}}=\nabla_{r_{M}^{\prime}} \circ\left(\lambda_{D} \otimes M\right)$,

(c5-3) $\left(\lambda_{D} \otimes M\right) \circ \nabla_{s_{M}}=\nabla_{s_{M}} \circ\left(\lambda_{D} \otimes M\right)$,

$\left(\mathrm{c5-4)}\left(M \otimes \lambda_{D}\right) \circ \nabla_{s_{M}^{\prime}}=\nabla_{s_{M}^{\prime}} \circ\left(M \otimes \lambda_{D}\right)\right.$.

Remark 2.7 In view of Definition 2.6 it follows that if $M=D$ is a WBHA in $\mathcal{C}$, the associated weak YangBaxter operator $t_{D, D}$ is an example of $(D, D)$-WO with $r_{M}=s_{M}=t_{D, D}$ and $r_{M}^{\prime}=s_{M}^{\prime}=t_{D, D}^{\prime}$; the claim remaining true if we take $t_{D, D}^{\prime}$ instead of $t_{D, D}$ and vice versa. Of course if $(\mathcal{C}, \otimes, c)$ is a braided monoidal category and $D$ is a WBHA in $\mathcal{C}$ with $t_{D, D}=c_{D, D}$ then $\left(c_{M, D}, c_{M, D}^{-1}, c_{D, M}, c_{D, M}^{-1}\right)$ and $\left(c_{D, M}^{-1}, c_{D, M}, c_{M, D}^{-1}, c_{M, D}\right)$ are both examples of $(M, D)$-WO for any object $M$ of $\mathcal{C}$.

Notice also that $\left(r_{M}, r_{M}^{\prime}, s_{M}, s_{M}^{\prime}\right)$ constitutes an $(M, D)$-WO iff so does $\left(s_{M}^{\prime}, s_{M}, r_{M}^{\prime}, r_{M}\right)$.

2.8. Formal properties of weak operators. Let $D$ be a WBHA in $\mathcal{C}$ and let $M$ be any object of the category such that $\left(r_{M}, r_{M}^{\prime}, s_{M}, s_{M}^{\prime}\right)$ constitutes an $(M, D)$-WO. We recall some properties about weak operators. As we have said in the introduction, the proofs have been given in [4].

$$
\begin{aligned}
& \text { The morphisms } \nabla_{r_{M}}, \nabla_{r_{M}^{\prime}}, \nabla_{s_{M}} \text { and } \nabla_{s_{M}^{\prime}} \text { are idempotent. } \\
& r_{M}=\nabla_{r_{M}^{\prime}} \circ r_{M}=r_{M} \circ \nabla_{r_{M}}, \quad s_{M}=\nabla_{s_{M}^{\prime}} \circ s_{M}=s_{M} \circ \nabla_{s_{M}}, \\
& r_{M}^{\prime}=r_{M}^{\prime} \circ \nabla_{r_{M}^{\prime}}=\nabla_{r_{M}} \circ r_{M}^{\prime}, \quad s_{M}^{\prime}=s_{M}^{\prime} \circ \nabla_{s_{M}^{\prime}}=\nabla_{s_{M}} \circ s_{M}^{\prime} .
\end{aligned}
$$

It holds that:

$$
\begin{aligned}
& \left(r_{M} \otimes D\right) \circ\left(M \otimes t_{D, D}\right) \circ\left(r_{M}^{\prime} \otimes D\right)=\left(D \otimes r_{M}^{\prime}\right) \circ\left(t_{D, D} \otimes M\right) \circ\left(D \otimes r_{M}\right), \\
& \left(D \otimes s_{M}\right) \circ\left(t_{D, D} \otimes M\right) \circ\left(D \otimes s_{M}^{\prime}\right)=\left(s_{M}^{\prime} \otimes D\right) \circ\left(M \otimes t_{D, D}\right) \circ\left(s_{M} \otimes D\right),
\end{aligned}
$$

and these two equalities remain true if we change $t_{D, D}$ by $t_{D, D}^{\prime}$.

Moreover, the following relations are also verified:

$$
\begin{aligned}
& \left(r_{M} \otimes D\right) \circ\left(M \otimes t_{D, D}\right) \circ\left(s_{M} \otimes D\right)=\left(D \otimes s_{M}\right) \circ\left(t_{D, D} \otimes M\right) \circ\left(D \otimes r_{M}\right), \\
& \left(s_{M}^{\prime} \otimes D\right) \circ\left(M \otimes t_{D, D}^{\prime}\right) \circ\left(r_{M}^{\prime} \otimes D\right)=\left(D \otimes r_{M}^{\prime}\right) \circ\left(t_{D, D}^{\prime} \otimes M\right) \circ\left(D \otimes s_{M}^{\prime}\right) .
\end{aligned}
$$

Generalizing the behavior of weak Yang-Baxter operators, it holds that:

$$
\begin{aligned}
\left(r_{M} \otimes D\right) \circ\left(M \otimes\left(\delta_{D} \circ \eta_{D}\right)\right) & =\left(D \otimes r_{M}^{\prime}\right) \circ\left(\left(\delta_{D} \circ \eta_{D}\right) \otimes M\right), \\
\left(\left(\varepsilon_{D} \circ \mu_{D}\right) \otimes M\right) \circ\left(D \otimes r_{M}\right) & =\left(M \otimes\left(\varepsilon_{D} \circ \mu_{D}\right)\right) \circ\left(r_{M}^{\prime} \otimes D\right), \\
\left(s_{M}^{\prime} \otimes D\right) \circ\left(M \otimes\left(\delta_{D} \circ \eta_{D}\right)\right) & =\left(D \otimes s_{M}\right) \circ\left(\left(\delta_{D} \circ \eta_{D}\right) \otimes M\right), \\
\left(\left(\varepsilon_{D} \circ \mu_{D}\right) \otimes M\right) \circ\left(D \otimes s_{M}^{\prime}\right) & =\left(M \otimes\left(\varepsilon_{D} \circ \mu_{D}\right)\right) \circ\left(s_{M} \otimes D\right) .
\end{aligned}
$$

If $M$ is any object in the category such that $\left(r_{M}, r_{M}^{\prime}, s_{M}, s_{M}^{\prime}\right)$ is an $(M, D)-\mathrm{WO}$, it holds that:

$$
\begin{array}{cc}
\left(\lambda_{D} \otimes M\right) \circ r_{M}=r_{M} \circ\left(M \otimes \lambda_{D}\right), & \left(M \otimes \lambda_{D}\right) \circ r_{M}^{\prime}=r_{M}^{\prime} \circ\left(\lambda_{D} \otimes M\right), \\
\left(\lambda_{D} \otimes M\right) \circ s_{M}^{\prime}=s_{M}^{\prime} \circ\left(M \otimes \lambda_{D}\right), & \left(M \otimes \lambda_{D}\right) \circ s_{M}=s_{M} \circ\left(\lambda_{D} \otimes M\right) .
\end{array}
$$

If $\lambda_{D}$ is an isomorphism, all the corresponding equalities obtained writing $\lambda_{D}^{-1}$ instead of $\lambda_{D}$ are also verified. As a consequence we have that

$$
\begin{gathered}
\left(\Pi_{D}^{L} \otimes M\right) \circ r_{M}=r_{M} \circ\left(M \otimes \Pi_{D}^{L}\right), r_{M}^{\prime} \circ\left(\Pi_{D}^{L} \otimes M\right)=\left(M \otimes \Pi_{D}^{L}\right) \circ r_{M}^{\prime}, \\
\left(\Pi_{D}^{L} \otimes M\right) \circ s_{M}^{\prime}=s_{M}^{\prime} \circ\left(M \otimes \Pi_{D}^{L}\right), s_{M} \circ\left(\Pi_{D}^{L} \otimes M\right)=\left(M \otimes \Pi_{D}^{L}\right) \circ s_{M},
\end{gathered}
$$

and the analogous equalities writing either $\Pi_{D}^{R}, \bar{\Pi}_{D}^{L}$, or $\bar{\Pi}_{D}^{R}$ instead of $\Pi_{D}^{L}$. 
Finally, if the antipode is an isomorphism, the following identities hold:

$$
\begin{aligned}
& \nabla_{r_{M}}=\left(M \otimes\left(\mu_{D} \circ t_{D, D}\right)\right) \circ\left(r_{M}^{\prime} \otimes D\right) \circ\left(\eta_{D} \otimes M \otimes D\right), \\
& \nabla_{r_{M}}=\left(\varepsilon_{D} \otimes M \otimes D\right) \circ\left(r_{M} \otimes D\right) \circ\left(M \otimes\left(t_{D, D} \circ \delta_{D}\right)\right), \\
& \nabla_{r_{M}^{\prime}}=\left(\left(\mu_{D} \circ t_{D, D}\right) \otimes M\right) \circ\left(D \otimes r_{M}\right) \circ\left(D \otimes M \otimes \eta_{D}\right), \\
& \nabla_{r_{M}^{\prime}}=\left(D \otimes M \otimes \varepsilon_{D}\right) \circ\left(D \otimes r_{M}^{\prime}\right) \circ\left(\left(t_{D, D} \circ \delta_{D}\right) \otimes M\right), \\
& \nabla_{s_{M}}=\left(\left(\mu_{D} \circ t_{D, D}\right) \otimes M\right) \circ\left(D \otimes s_{M}^{\prime}\right) \circ\left(D \otimes M \otimes \eta_{D}\right), \\
& \nabla_{s_{M}}=\left(D \otimes M \otimes \varepsilon_{D}\right) \circ\left(D \otimes s_{M}\right) \circ\left(\left(t_{D, D} \circ \delta_{D}\right) \otimes M\right), \\
& \nabla_{s_{M}^{\prime}}=\left(M \otimes\left(\mu_{D} \circ t_{D, D}\right)\right) \circ\left(s_{M} \otimes D\right) \circ\left(\eta_{D} \otimes M \otimes D\right), \\
& \nabla_{s_{M}^{\prime}}=\left(\varepsilon_{D} \otimes M \otimes D\right) \circ\left(s_{M}^{\prime} \otimes D\right) \circ\left(M \otimes\left(t_{D, D} \circ \delta_{D}\right)\right),
\end{aligned}
$$

and the corresponding equalities changing $t_{D, D}$ by $t_{D, D}^{\prime}$ are also satisfied.

The below results about WBHA's can be demonstrated arguing similarly than in the analogous well known standard results about weak Hopf algebras, incorporating the suitable application of conditions (b1) and (b2) of WBHA's.

Proposition 2.9 Let $\left(D, \eta_{D}, \mu_{D}, \varepsilon_{D}, \delta_{D}\right)$ a WBHA with associated weak Yang-Baxter operator $t_{D, D}$ and such that its antipode $\lambda_{D}$ is an isomorphism.

(i) $D^{\mathrm{op}}=\left(D, \eta_{D^{\mathrm{op}}}=\eta_{D}, \mu_{D^{\mathrm{op}}}=\mu_{D} \circ t_{D, D}^{\prime}, \varepsilon_{D^{\mathrm{op}}}=\varepsilon_{D}, \delta_{D^{\mathrm{op}}}=\delta_{D}\right)$ with associated weak Yang-Baxter operator $t_{D^{\mathrm{op}}, D^{\mathrm{op}}}=t_{D, D}^{\prime}$ and antipode $\lambda_{D^{\mathrm{op}}}=\lambda_{D}^{-1}$ is a WBHA.

(ii) $D^{\text {coop }}=\left(D, \eta_{D^{\text {coop }}}=\eta_{D}, \mu_{D^{\text {coop }}}=\mu_{D}, \varepsilon_{D^{\text {coop }}}=\varepsilon_{D}, \delta_{D^{\text {coop }}}=t_{D, D}^{\prime} \circ \delta_{D}\right)$ with associated weak Yang-Baxter operator $t_{D^{\text {coop }}, D^{\text {coop }}}=t_{D, D}^{\prime}$ and antipode $\lambda_{D^{\text {coop }}}=\lambda_{D}^{-1}$ is a WBHA.

(iii) $D^{\text {op }}=\left(D, \eta_{D^{\text {opo }} \text { coop }}=\eta_{D}, \mu_{D^{\text {op }} \text { coop }}=\mu_{D} \circ t_{D, D}^{\prime}, \varepsilon_{D^{\text {op }} \text { coop }}=\varepsilon_{D}, \delta_{D^{\text {op }} \text { cop }}=t_{D, D} \circ \delta_{D}\right)$ with

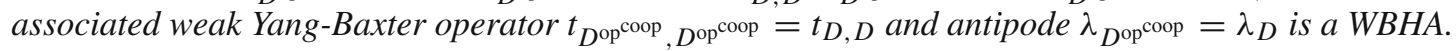

(iv) $D^{\text {coop }}=\left(D, \eta_{D^{\text {coop }}}=\eta_{D}, \mu_{D^{\text {copp }}}^{\text {op }}=\mu_{D} \circ t_{D, D}, \varepsilon_{D^{\text {coop }}}{ }^{\mathrm{op}}=\varepsilon_{D}, \delta_{D^{\text {coop }}}=t_{D, D}^{\prime} \circ \delta_{D}\right)$ with associated weak Yang-Baxter operator $t_{D^{\text {coop }}}{ }^{\mathrm{op}}, D^{\text {coop }}{ }^{\mathrm{op}}=t_{D, D}$ and antipode $\lambda_{D^{\text {coop }}}{ }^{\mathrm{op}}=\lambda_{D}$ is a WBHA.

This being the situation, in virtue of equalities (25)-(32) we conclude that given a WBHA $D$ with invertible antipode and an $(M, D)$-WO, all the morphisms $\nabla_{r_{M}}, \nabla_{r_{M}^{\prime}}, \nabla_{s_{M}}$ and $\nabla_{s_{M}^{\prime}}$ are equal for $D^{\mathrm{op}}, D^{\text {coop }}$ and $D$, so a given weak operator automatically provides weak operator structures for all the various WBHA structures arising naturally when twisting the (co)multiplication from $D$. More precisely:

Proposition 2.10 Let D be a WBHA such that the antipode is an isomorphism and let $M$ be any object of $\mathcal{C}$. Then the following statements are equivalent:

(i) $\left(r_{M}, r_{M}^{\prime}, s_{M}, s_{M}^{\prime}\right)$ is an $(M, D)-W O$.

(ii) $\left(s_{M}^{\prime}, s_{M}, r_{M}^{\prime}, r_{M}\right)$ is an $\left(M, D^{\text {op }}\right)-W O$.

(iii) $\left(s_{M}^{\prime}, s_{M}, r_{M}^{\prime}, r_{M}\right)$ is an $\left(M, D^{\text {coop }}\right)-W O$.

As a consequence we get the corresponding equivalences for $D^{\mathrm{op}}{ }^{\text {coop }}$ and $D^{\text {coop }}{ }^{\text {op }}$.

Proof Straightforward.

When dealing with an $(M, D)$-WO, if the object $M$ is equipped with an algebraic structure it enriches that of weak operator. We will explain how if $M$ is endowed with a $D$-(co)module structure, then the existence of an $(M, D)$-WO allows to obtain many other (co)-module structures in a systematic way.

Let us recall the notion of weak operator compatible with a (co)module structure of $M$.

Definition 2.11 Let $D$ be a WBHA, $M$ an object of $\mathcal{C}$ and $\left(r_{M}, r_{M}^{\prime}, s_{M}, s_{M}^{\prime}\right)$ an $(M, D)$-WO.

(i) If $\left(M, \varphi_{M}\right)$ is a left $D$-module, the $(M, D)$-WO is said to be compatible with the $D$-module structure provided that it satisfies:

$(\mathrm{i}-1) r_{M} \circ\left(\varphi_{M} \otimes D\right)=\left(D \otimes \varphi_{M}\right) \circ\left(t_{D, D} \otimes M\right) \circ\left(D \otimes r_{M}\right)$,

(i-2) $r_{M}^{\prime} \circ\left(D \otimes \varphi_{M}\right)=\left(\varphi_{M} \otimes D\right) \circ\left(D \otimes r_{M}^{\prime}\right) \circ\left(t_{D, D}^{\prime} \otimes M\right)$,

$(\mathrm{i}-3) s_{M}^{\prime} \circ\left(\varphi_{M} \otimes D\right)=\left(D \otimes \varphi_{M}\right) \circ\left(t_{D, D}^{\prime} \otimes M\right) \circ\left(D \otimes s_{M}^{\prime}\right)$, 
(i-4) $s_{M} \circ\left(D \otimes \varphi_{M}\right)=\left(\varphi_{M} \otimes D\right) \circ\left(D \otimes s_{M}\right) \circ\left(t_{D, D} \otimes M\right)$.

(ii) If $\left(M, \varrho_{M}\right)$ is a left $D$-comodule, the $(M, D)$-WO is said to be compatible with the $D$-comodule structure provided that it satisfies:

(ii-1) $\left(D \otimes \varrho_{M}\right) \circ r_{M}=\left(t_{D, D} \otimes M\right) \circ\left(D \otimes r_{M}\right) \circ\left(\varrho_{M} \otimes D\right)$,

(ii-2) $\left(\varrho_{M} \otimes D\right) \circ r_{M}^{\prime}=\left(D \otimes r_{M}^{\prime}\right) \circ\left(t_{D, D}^{\prime} \otimes M\right) \circ\left(D \otimes \varrho_{M}\right)$,

(ii-3) $\left(D \otimes \varrho_{M}\right) \circ s_{M}^{\prime}=\left(t_{D, D}^{\prime} \otimes M\right) \circ\left(D \otimes s_{M}^{\prime}\right) \circ\left(\varrho_{M} \otimes D\right)$,

(ii-4) $\left(\varrho_{M} \otimes D\right) \circ s_{M}=\left(D \otimes s_{M}\right) \circ\left(t_{D, D} \otimes M\right) \circ\left(D \otimes \varrho_{M}\right)$.

By doing the suitable changes one defines the compatibility for right structures.

Notice that in the particular case of $\mathcal{C}$ being a braided category with braiding $c$ the conditions trivialize because of $t_{D, D}=c_{D, D}, t_{D, D}^{\prime}=c_{D, D}^{-1}, r_{M}=c_{M, D}, r_{M}^{\prime}=c_{M, D}^{-1}, s_{M}=c_{D, M}$ and $s_{M}^{\prime}=c_{D, M}^{-1}$. Then in that context the compatibility is not a restriction.

Lemma 2.12 Let $D$ be a WBHA, $M$ an object of $|\mathcal{C}|$ and $\left(r_{M}, r_{M}^{\prime}, s_{M}, s_{M}^{\prime}\right)$ an $(M, D)-W O$. It holds that:

(i) If $\left(M, \varphi_{M}\right)$ is a left D-module then $\varphi_{M}=\varphi_{M} \circ \nabla_{r_{M}^{\prime}}$ iff $\varphi_{M} \circ r_{M} \circ\left(M \otimes \eta_{D}\right)=i d_{M}$.

(ii) If $\left(M, \psi_{M}\right)$ is a right $D$-module then $\psi_{M}=\psi_{M} \circ \nabla_{r_{M}}$ iff $\psi_{M} \circ r_{M}^{\prime} \circ\left(\eta_{D} \otimes M\right)=i d_{M}$.

(iii) If $\left(M, \varrho_{M}\right)$ is a left $D$-comodule then $\varrho_{M}=\nabla_{r_{M}^{\prime}} \circ \varrho_{M}$ iff $\left(M \otimes \varepsilon_{D}\right) \circ r_{M}^{\prime} \circ \varrho_{M}=i d_{M}$.

(iv) If $\left(M, \rho_{M}\right)$ is a right D-comodule then $\rho_{M}=\nabla_{r_{M}} \circ \rho_{M}$ iff $\left(\varepsilon_{D} \otimes M\right) \circ r_{M} \circ \rho_{M}=i d_{M}$.

The analogous equalities changing either $s_{M}$ by $r_{M}^{\prime}$ and $\nabla_{r_{M}}$ by $\nabla_{s_{M}^{\prime}}$, or $s_{M}^{\prime}$ by $r_{M}$ and $\nabla_{r_{M}^{\prime}}$ by $\nabla_{s_{M}}$ also hold.

Proof Straightforward.

The next propositions make explicit how weak operators allow to generate new (co)module structures on $M$ preserving compatibility.

Proposition 2.13 Let $D$ be a WBHA with invertible antipode, $\left(M, \varphi_{M}\right)$ a left $D$-module and $\left(r_{M}, r_{M}^{\prime}, s_{M}, s_{M}^{\prime}\right)$ an $(M, D)-W O$ compatible with the module structure.

(i) If in addition it holds that

$$
\varphi_{M} \circ r_{M} \circ\left(M \otimes \eta_{D}\right)=i d_{M}
$$

then

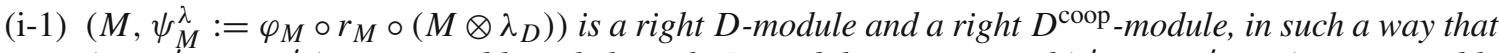
$\left(r_{M}, r_{M}^{\prime}, s_{M}, s_{M}^{\prime}\right)$ is compatible with the right $D$-module structure and $\left(s_{M}^{\prime}, s_{M}, r_{M}^{\prime}, r_{M}\right)$ is compatible with the right $D^{\mathrm{coop}}$-module structure.

(i-2) $\left(M, \psi_{M}:=\varphi_{M} \circ r_{M}\right)$ is a right- $D^{\text {coopop }}$-module and $\left(r_{M}, r_{M}^{\prime}, s_{M}, s_{M}^{\prime}\right)$ is compatible with the $D^{\text {coopop }}$. module structure.

(ii) If in addition it holds that

$$
\varphi_{M} \circ s_{M}^{\prime} \circ\left(M \otimes \eta_{D}\right)=i d_{M}
$$

then

(ii-1) $\left(M, \psi_{M}^{\lambda^{-1}}:=\varphi_{M} \circ s_{M}^{\prime} \circ\left(M \otimes \lambda_{D}^{-1}\right)\right)$ is a right $D$-module and a right $D^{\text {coop }}$-module, in such a way that $\left(r_{M}, r_{M}^{\prime}, s_{M}, s_{M}^{\prime}\right)$ is compatible with the right D-module structure and $\left(s_{M}^{\prime}, s_{M}, r_{M}^{\prime}, r_{M}\right)$ is compatible with the $D^{\mathrm{coop}}$-module structure.

(ii-2) $\left(M, \psi_{M}^{\prime}:=\varphi_{M} \circ s_{M}^{\prime}\right)$ is a right $D^{\mathrm{op}}$-module and a $D^{\mathrm{coop}{ }^{\mathrm{op}}}$-module in such a way that $\left(s_{M}^{\prime}, s_{M}, r_{M}^{\prime}, r_{M}\right)$ is compatible with the $D^{\mathrm{op}}$-module structure while $\left(r_{M}, r_{M}^{\prime}, s_{M}, s_{M}^{\prime}\right)$ is compatible with the $D^{\mathrm{coop}^{\mathrm{op}}-}$ module structure.

Proof We prove Part (i). As $\lambda_{D} \circ \eta_{D}=\eta_{D}$ it is obvious that $\psi_{M}^{\lambda} \circ\left(M \otimes \eta_{D}\right)=i d_{M}$. Now, by compatibility, conditions (c1), (c4) and (i-1) of Definition 2.11, and the fact that $\mu_{D} \circ t_{D, D} \circ\left(\lambda_{D} \otimes \lambda_{D}\right)=\lambda_{D} \circ \mu_{D}$, we obtain

$$
\begin{aligned}
\psi_{M}^{\lambda} \circ\left(\psi_{M}^{\lambda} \otimes D\right) & =\varphi_{M} \circ\left(D \otimes \varphi_{M}\right) \circ\left(t_{D, D} \otimes M\right) \circ\left(D \otimes r_{M}\right) \circ\left(r_{M} \otimes D\right) \circ\left(M \otimes \lambda_{D} \otimes \lambda_{D}\right) \\
& =\varphi_{M} \circ r_{M} \circ\left(M \otimes\left(\mu_{D} \circ t_{D, D} \circ\left(\lambda_{D} \otimes \lambda_{D}\right)\right)\right) \\
& =\psi_{M}^{\lambda} \circ\left(M \otimes \mu_{D}\right),
\end{aligned}
$$


so $\left(M, \psi_{M}^{\lambda}\right)$ is a right $D$-module and hence a right $D^{\text {coop }}$-module. By the same argument we conclude that $\left(M, \psi_{M}\right)$ is a right $D^{\text {coop }}{ }^{\text {op }}$-module.

Compatibility can be proved using compatibility with $\varphi_{M}$, equalities (21), (13)-(16), (8) and (9), and conditions (c1-1) and (c2-4).

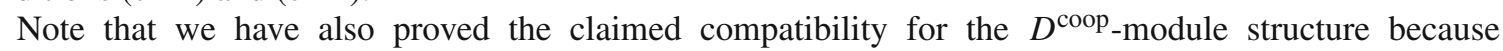
$t_{D^{\text {coop }}, D^{\text {coop }}}=t_{D, D}^{\prime}$.

The proof of the other statements of the proposition follows the same pattern, just remembering that $\mu_{D} \circ t_{D, D}^{\prime} \circ\left(\lambda_{D}^{-1} \otimes \lambda_{D}^{-1}\right)=\lambda_{D}^{-1} \circ \mu_{D}$ (see Proposition 2.20 of [2]).

Arguing in a similar way we obtain:

Proposition 2.14 Let D be a WBHA with invertible antipode, $\left(M, \psi_{M}\right)$ a right D-module and $\left(r_{M}, r_{M}^{\prime}, s_{M}, s_{M}^{\prime}\right)$ an $(M, D)$-WO compatible with the module structure.

(i) If in addition it holds that

$$
\psi_{M} \circ s_{M} \circ\left(\eta_{D} \otimes M\right)=i d_{M}
$$

then

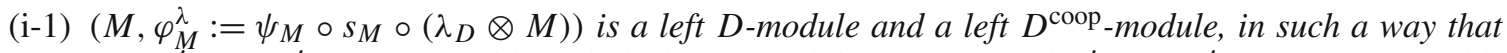
$\left(r_{M}, r_{M}^{\prime}, s_{M}, s_{M}^{\prime}\right)$ is compatible with the left D-module structure and $\left(s_{M}^{\prime}, s_{M}, r_{M}^{\prime}, r_{M}\right)$ is compatible with the left $D^{\mathrm{coop}}$-module structure.

(i-2) $\left(M, \varphi_{M}:=\psi_{M} \circ s_{M}\right)$ is a left $D^{\mathrm{coop}{ }^{\mathrm{op}}}$-module in such a way that $\left(r_{M}, r_{M}^{\prime}, s_{M}, s_{M}^{\prime}\right)$ is compatible with the $D^{\text {coop }}{ }^{\text {op }}$-module structure.

(ii) If in addition it holds that

$$
\psi_{M} \circ r_{M}^{\prime} \circ\left(\eta_{D} \otimes M\right)=i d_{M}
$$

then

(ii-1) $\left(M, \varphi_{M}^{\lambda^{-1}}:=\psi_{M} \circ r_{M}^{\prime} \circ\left(\lambda_{D}^{-1} \otimes M\right)\right)$ is a left D-module and a left $D^{\text {coop }}$-module, in such a way that $\left(r_{M}, r_{M}^{\prime}, s_{M}, s_{M}^{\prime}\right)$ is compatible with the left D-module structure and $\left(s_{M}^{\prime}, s_{M}, r_{M}^{\prime}, r_{M}\right)$ is compatible with the $D^{\text {coop }}$-module structure.

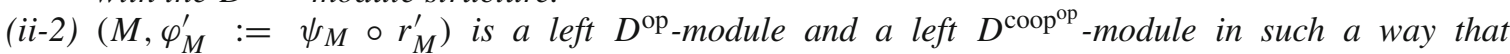
$\left(s_{M}^{\prime}, s_{M}, r_{M}^{\prime}, r_{M}\right)$ is compatible with the $D^{\mathrm{op}}$-module structure while $\left(r_{M}, r_{M}^{\prime}, s_{M}, s_{M}^{\prime}\right)$ is compatible with the $D^{\text {coop }}{ }^{\text {op }}$-module structure.

Doing the suitable changes we get the expected results for comodules:

Proposition 2.15 Let $D$ be a WBHA with invertible antipode, $\left(M, \varrho_{M}\right)$ a left D-comodule and $\left(r_{M}, r_{M}^{\prime}, s_{M}\right.$, $\left.s_{M}^{\prime}\right)$ an $(M, D)$-WO compatible with the comodule structure.

(i) If in addition it holds that

$$
\left(M \otimes \varepsilon_{D}\right) \circ s_{M} \circ \varrho_{M}=i d_{M}
$$

then

(i-1) $\left(M, \rho_{M}^{\lambda}:=\left(M \otimes \lambda_{D}\right) \circ s_{M} \circ \varrho_{M}\right)$ is a right D-comodule and a right $D^{\mathrm{op}}$-comodule, in such a way that $\left(r_{M}, r_{M}^{\prime}, s_{M}, s_{M}^{\prime}\right)$ is compatible with the new D-comodule structure and $\left(s_{M}^{\prime}, s_{M}, r_{M}^{\prime}, r_{M}\right)$ is compatible with the $D^{\mathrm{op}}$-comodule structure.

(i-2) $\left(M, \rho_{M}:=s_{M} \circ \varrho_{M}\right)$ is a right $D^{\text {coop }}{ }^{\mathrm{op}}$-comodule and $\left(r_{M}, r_{M}^{\prime}, s_{M}, s_{M}^{\prime}\right)$ is compatible with the $D^{\text {coop }}{ }^{\text {op }}$-comodule structure.

(ii) If in addition it holds that

$$
\left(M \otimes \varepsilon_{D}\right) \circ r_{M}^{\prime} \circ \varrho_{M}=i d_{M}
$$

then

(ii-1) $\left(M, \rho_{M}^{\lambda^{-1}}:=\left(M \otimes \lambda_{D}^{-1}\right) \circ r_{M}^{\prime} \circ \varrho_{M}\right)$ is a right $D$-comodule and a right $D^{\mathrm{op}}$-comodule, in such a way that $\left(r_{M}, r_{M}^{\prime}, s_{M}, s_{M}^{\prime}\right)$ is compatible with the right $D$-comodule structure and $\left(s_{M}^{\prime}, s_{M}, r_{M}^{\prime}, r_{M}\right)$ is compatible with the $D^{\mathrm{op}}$-comodule structure. 
(ii-2) $\left(M, \rho_{M}^{\prime}:=r_{M}^{\prime} \circ \varrho_{M}\right)$ is a right $D^{\text {coop }}$-comodule and a $D^{\text {coop }}{ }^{\text {op }}$-comodule in such a way that $\left(r_{M}, r_{M}^{\prime}, s_{M}, s_{M}^{\prime}\right)$ is compatible with the $D^{\text {coop }}{ }^{\text {op }}$-comodule structure while $\left(s_{M}^{\prime}, s_{M}, r_{M}^{\prime}, r_{M}\right)$ is compatible with the $D^{\text {coop }}$-comodule structure.

Proposition 2.16 Let D be a WBHA with invertible antipode, $\left(M, \rho_{M}\right)$ a right $D$-comodule and $\left(r_{M}, r_{M}^{\prime}, s_{M}\right.$, $\left.s_{M}^{\prime}\right)$ an $(M, D)$-WO compatible with the comodule structure.

(i) If in addition it holds that

$$
\left(\varepsilon_{D} \otimes M\right) \circ r_{M} \circ \rho_{M}=i d_{M}
$$

then

(i-1) $\left(M, \varrho_{M}^{\lambda}:=\left(\lambda_{D} \otimes M\right) \circ r_{M} \circ \rho_{M}\right)$ is a left D-comodule and a left $D^{\mathrm{op}}$-comodule, in such a way that $\left(r_{M}, r_{M}^{\prime}, s_{M}, s_{M}^{\prime}\right)$ is compatible with the left D-comodule structure and $\left(s_{M}^{\prime}, s_{M}, r_{M}^{\prime}, r_{M}\right)$ is compatible with the $D^{\mathrm{op}}$-comodule structure.

(i-2) $\left(M, \varrho_{M}:=r_{M} \circ \rho_{M}\right)$ is a left $D^{\mathrm{coop}{ }^{\mathrm{op}}}$-comodule and $\left(r_{M}, r_{M}^{\prime}, s_{M}, s_{M}^{\prime}\right)$ is compatible with the $D^{\mathrm{coop}}$ comodule structure.

(ii) If in addition it holds that

$$
\left(\varepsilon_{D} \otimes M\right) \circ s_{M}^{\prime} \circ \rho_{M}=i d_{M}
$$

then

(ii-1) $\left(M, \varrho_{M}^{\lambda^{-1}}:=\left(\lambda_{D}^{-1} \otimes M\right) \circ s_{M}^{\prime} \circ \rho_{M}\right)$ is a left D-comodule and a left $D^{\mathrm{op}}$-comodule, in such a way that $\left(r_{M}, r_{M}^{\prime}, s_{M}, s_{M}^{\prime}\right)$ is compatible with the new D-comodule structure and $\left(s_{M}^{\prime}, s_{M}, r_{M}^{\prime}, r_{M}\right)$ is compatible with the $D^{\mathrm{op}}$-comodule structure.

(ii-2) $\left(M, \varrho_{M}^{\prime}:=s_{M}^{\prime} \circ \rho_{M}\right)$ is a left $D^{\text {coop }}$-comodule and a $D^{\text {coop }}{ }_{\text {-comodule in such a way that }}$ $\left(r_{M}, r_{M}^{\prime}, s_{M}, s_{M}^{\prime}\right)$ is compatible with the $D^{\text {coop }}{ }^{\text {op }}$-comodule structure while $\left(s_{M}^{\prime}, s_{M}, r_{M}^{\prime}, r_{M}\right)$ is compatible with the $D^{\text {coop }}$-comodule structure.

\section{Equivalences between Yetter-Drinfeld categories}

This section is devoted to the study of the several Yetter-Drinfeld categories that arise naturally starting from a given arbitrary WBHA $D$, focussing on the various relations between them. The framework we deal with is the general context of an ambient monoidal category $\mathcal{C}$ that is not assumed to be equipped with a braiding, considering the (co)module structures defined over a WBHA. In this situation, the first difficulty consists on giving suitable definitions of Yetter-Drinfeld modules such that we recover those introduced in [15] when we restrict to the particular case of modules over a weak Hopf algebra in a symmetric category, providing the basis needed to develop the theory of Yetter-Drinfeld categories in a general monoidal context.

The following notion was introduced in [4]:

Definition 3.1 Let $D$ be a WBHA. We say that $\left(M, \varphi_{M}, \varrho_{M}\right)$ is a left-left Yetter-Drinfeld module over $D$ if $\left(M, \varphi_{M}\right)$ is a left $D$-module, $\left(M, \varrho_{M}\right)$ is a left $D$-comodule and:

$(\mathrm{yd} 1-11) \varrho_{M}=\left(\mu_{D} \otimes \varphi_{M}\right) \circ\left(D \otimes t_{D, D} \otimes M\right) \circ\left(\delta_{D} \otimes \varrho_{M}\right) \circ\left(\eta_{D} \otimes M\right)$.

(yd2-11) There exists $\left(r_{M}, r_{M}^{\prime}, s_{M}, s_{M}^{\prime}\right)$ an $(M, D)$-WO compatible with the (co)module structures of $M$, such that

$$
\begin{aligned}
& \left(\mu_{D} \otimes \varphi_{M}\right) \circ\left(D \otimes t_{D, D} \otimes M\right) \circ\left(\delta_{D} \otimes \varrho_{M}\right) \\
& \quad=\left(\mu_{D} \otimes M\right) \circ\left(D \otimes r_{M}\right) \circ\left(\left(\varrho_{M} \circ \varphi_{M}\right) \otimes D\right) \circ\left(D \otimes s_{M}\right) \circ\left(\delta_{D} \otimes M\right) .
\end{aligned}
$$

Definition 3.2 Let $D$ be a WBHA. We say that $\left(M, \psi_{M}, \rho_{M}\right)$ is a right-right Yetter-Drinfeld module over $D$ if $\left(M, \psi_{M}\right)$ is a right $D$-module, $\left(M, \rho_{M}\right)$ is a right $D$-comodule and:

$(\mathrm{yd} 1-\mathrm{rr}) \rho_{M}=\left(\psi_{M} \otimes \mu_{D}\right) \circ\left(M \otimes t_{D, D} \otimes D\right) \circ\left(\rho_{M} \otimes \delta_{D}\right) \circ\left(M \otimes \eta_{D}\right)$.

(yd2-rr) There exists $\left(r_{M}, r_{M}^{\prime}, s_{M}, s_{M}^{\prime}\right)$ an $(M, D)$-WO compatible with the (co)module structures of $M$ such that

$$
\begin{aligned}
& \left(\psi_{M} \otimes \mu_{D}\right) \circ\left(M \otimes t_{D, D} \otimes D\right) \circ\left(\rho_{M} \otimes \delta_{D}\right) \\
& \quad=\left(M \otimes \mu_{D}\right) \circ\left(s_{M} \otimes D\right) \circ\left(D \otimes\left(\rho_{M} \circ \psi_{M}\right)\right) \circ\left(r_{M} \otimes D\right) \circ\left(M \otimes \delta_{D}\right) .
\end{aligned}
$$


Definition 3.3 Let $D$ be a WBHA. We say that $\left(M, \varphi_{M}, \rho_{M}\right)$ is a left-right Yetter-Drinfeld module over $D$ if $\left(M, \varphi_{M}\right)$ is a left $D$-module, $\left(M, \rho_{M}\right)$ is a right $D$-comodule and:

(yd1-lr) There exist $\left(r_{M}, r_{M}^{\prime}, s_{M}, s_{M}^{\prime}\right)$ an $(M, D)$-WO compatible with the (co)module structures of $M$ such that

$$
\rho_{M}=\left(\varphi_{M} \otimes \mu_{D}\right) \circ\left(D \otimes s_{M} \otimes D\right) \circ\left(\delta_{D} \otimes \rho_{M}\right) \circ\left(\eta_{D} \otimes M\right) .
$$

(yd2-lr) Taking the same $(M, D)$-WO required to exist in condition (yd1-lr) it holds that:

$$
\begin{aligned}
& \left(\varphi_{M} \otimes \mu_{D}\right) \circ\left(D \otimes s_{M} \otimes D\right) \circ\left(\delta_{D} \otimes \rho_{M}\right) \\
& \quad=\left(M \otimes\left(\mu_{D} \circ t_{D, D}^{\prime}\right)\right) \circ\left(r_{M}^{\prime} \otimes D\right) \circ\left(D \otimes\left(\rho_{M} \circ \varphi_{M}\right)\right) \circ\left(\delta_{D} \otimes M\right) .
\end{aligned}
$$

Definition 3.4 Let $D$ be a WBHA. We say that $\left(M, \psi_{M}, \varrho_{M}\right)$ is a right-left Yetter Drinfeld module over $D$ if $\left(M, \psi_{M}\right)$ is a right $D$-module, $\left(M, \varrho_{M}\right)$ is a left $D$-comodule and:

(yd1-rl) There exist $\left(r_{M}, r_{M}^{\prime}, s_{M}, s_{M}^{\prime}\right)$ an $(M, D)$-WO compatible with the (co)module structures of $M$ such that

$$
\varrho_{M}=\left(\mu_{D} \otimes \psi_{M}\right) \circ\left(D \otimes r_{M} \otimes D\right) \circ\left(\varrho_{M} \otimes \delta_{D}\right) \circ\left(M \otimes \eta_{D}\right) .
$$

(yd2-rl) Taking the same $(M, D)$-WO required to exist in condition (yd1-rl) it holds that:

$$
\left(\mu_{D} \otimes \psi_{M}\right) \circ\left(D \otimes r_{M} \otimes D\right) \circ\left(\varrho_{M} \otimes \delta_{D}\right)=\left(\left(\mu_{D} \circ t_{D, D}^{\prime}\right) \otimes M\right) \circ\left(D \otimes s_{M}^{\prime}\right) \circ\left(\left(\varrho_{M} \circ \psi_{M}\right) \otimes D\right) \circ\left(M \otimes \delta_{D}\right) .
$$

Remark 3.5 Note that if $\mathcal{C}$ is a braided category with braiding $c$, taking $t_{D, D}=c_{D, D}, t_{D, D}^{\prime}=c_{D, D}^{-1}$ then $\nabla_{D, D}=i d_{D \otimes D}$ and $D$ is a weak Hopf algebra in $\mathcal{C}$ (see [1] and [2]). Having into account that for any object $M$ in $\mathcal{C}$ the quadruple $\left(c_{M, D}, c_{M, D}^{-1}, c_{D, M}, c_{D, M}^{-1}\right)$ is an $(M, D)$-WO, we obtain the various definitions of Yetter-Drinfeld modules over a weak Hopf algebra $D$ in a braided category $\mathcal{C}$. Precisely:

(i) A left $D$-(co)module $\left(M, \varphi_{M}, \varrho_{M}\right)$ is a left-left Yetter-Drinfeld module over $D$ if it satisfies:

$\left(\right.$ yd1-11) $\varrho_{M}=\left(\mu_{D} \otimes \varphi_{M}\right) \circ\left(D \otimes c_{D, D} \otimes M\right) \circ\left(\delta_{D} \otimes \varrho_{M}\right) \circ\left(\eta_{D} \otimes M\right)$,

$(\mathrm{yd} 2-11)\left(\mu_{D} \otimes \varphi_{M}\right) \circ\left(D \otimes c_{D, D} \otimes M\right) \circ\left(\delta_{D} \otimes \varrho_{M}\right)$

$$
=\left(\mu_{D} \otimes M\right) \circ\left(D \otimes c_{M, D}\right) \circ\left(\left(\varrho_{M} \circ \varphi_{M}\right) \otimes D\right) \circ\left(D \otimes c_{D, M}\right) \circ\left(\delta_{D} \otimes M\right) .
$$

(ii) A right $D$-(co)module $\left(M, \psi_{M}, \rho_{M}\right)$ is a right-right Yetter-Drinfeld module over $D$ if it satisfies:

$\left(\right.$ yd1-rr) $\rho_{M}=\left(\psi_{M} \otimes \mu_{D}\right) \circ\left(M \otimes c_{D, D} \otimes D\right) \circ\left(\rho_{M} \otimes \delta_{D}\right) \circ\left(M \otimes \eta_{D}\right)$,

$(\mathrm{yd} 2-\mathrm{rr})\left(\psi_{M} \otimes \mu_{D}\right) \circ\left(M \otimes c_{D, D} \otimes D\right) \circ\left(\rho_{M} \otimes \delta_{D}\right)$ $=\left(M \otimes \mu_{D}\right) \circ\left(c_{D, M} \otimes D\right) \circ\left(D \otimes\left(\rho_{M} \circ \psi_{M}\right)\right) \circ\left(c_{M, D} \otimes D\right) \circ\left(M \otimes \delta_{D}\right)$.

(iii) A left $D$-module and right $D$-comodule $\left(M, \varphi_{M}, \rho_{M}\right)$ is a left-right Yetter-Drinfeld module over $D$ if it satisfies:

$\left(\right.$ yd1-lr) $\rho_{M}=\left(\varphi_{M} \otimes \mu_{D}\right) \circ\left(D \otimes c_{D, M} \otimes D\right) \circ\left(\delta_{D} \otimes \rho_{M}\right) \circ\left(\eta_{D} \otimes M\right)$,

$($ yd2-lr $)\left(\varphi_{M} \otimes \mu_{D}\right) \circ\left(D \otimes c_{D, M} \otimes D\right) \circ\left(\delta_{D} \otimes \rho_{M}\right)$

$$
=\left(M \otimes\left(\mu_{D} \circ c_{D, D}^{-1}\right)\right) \circ\left(c_{M, D}^{-1} \otimes D\right) \circ\left(D \otimes\left(\rho_{M} \circ \varphi_{M}\right)\right) \circ\left(\delta_{D} \otimes M\right) .
$$

(iv) A right $D$-module and left $D$-comodule $\left(M, \psi_{M}, \varrho_{M}\right)$ is a right-left Yetter Drinfeld module over $D$ if it satisfies:

$($ yd1-rl $) \varrho_{M}=\left(\mu_{D} \otimes \psi_{M}\right) \circ\left(D \otimes c_{M, D} \otimes D\right) \circ\left(\varrho_{M} \otimes \delta_{D}\right) \circ\left(M \otimes \eta_{D}\right)$,

$(\mathrm{yd} 2-\mathrm{rl})\left(\mu_{D} \otimes \psi_{M}\right) \circ\left(D \otimes c_{M, D} \otimes D\right) \circ\left(\varrho_{M} \otimes \delta_{D}\right)$

$$
=\left(\left(\mu_{D} \circ c_{D, D}^{-1}\right) \otimes M\right) \circ\left(D \otimes c_{D, M}^{-1}\right) \circ\left(\left(\varrho_{M} \circ \psi_{M}\right) \otimes D\right) \circ\left(M \otimes \delta_{D}\right) .
$$

Obviously, when the ambient category $\mathcal{C}$ is symmetric (e.g., the braiding $c$ is such that $c_{N, M} \circ c_{M, N}=i d_{M \otimes N}$ for all $M, N$ in $\mathcal{C}$ ), we also recover the classic definitions of Yetter-Drinfeld modules introduced in [15] in the context of Hopf algebras and generalized in [6] to the context of weak Hopf algebras.

Definition 3.6 Let $\left(M, \varphi_{M}, \varrho_{M}\right)$ and $\left(N, \varphi_{N}, \varrho_{N}\right)$ be left-left Yetter-Drinfeld modules with associated weak operators $\left(r_{M}, r_{M}^{\prime}, s_{M}, s_{M}^{\prime}\right)$ and $\left(r_{N}, r_{N}^{\prime}, s_{N}, s_{N}^{\prime}\right)$ respectively. It is said that $f: M \rightarrow N$ is a morphism of left-left Yetter-Drinfeld modules if:

(e1) $f$ is a left (co)module morphism.

(e2) $r_{N} \circ(f \otimes D)=(D \otimes f) \circ r_{M}, \quad s_{N} \circ(D \otimes f)=(f \otimes D) \circ s_{M}$. 
The definitions for the morphisms of right-right, right-left and left-right Yetter-Drinfeld modules are stated analogously.

Remark 3.7 In the last definition, the verification of condition (e2) for $r_{M}$ is equivalent to its verification for $r_{M}^{\prime}$, and the same happens with $s_{M}$ and $s_{M}^{\prime}$.

As the identity morphism $i d_{M}$ verifies the above conditions for any object $M$, it can be introduced the following:

Definition 3.8 The category whose objects are those left-left Yetter-Drinfeld modules and whose morphisms are those in the conditions of Definition 3.6 will be denoted also by ${ }_{D}^{D} \mathcal{Y} \mathcal{D}$. The categories $\mathcal{Y} \mathcal{D}_{D}^{D},{ }_{D} \mathcal{Y} \mathcal{D}^{D}$ and ${ }^{D} \mathcal{Y} \mathcal{D}_{D}$ are defined analogously.

Remark 3.9 In a similar way to the classical case, conditions (yd1-11) and (yd2-11) in Definition 3.1 can also be restated as follows: Suppose that $\left(M, \varphi_{M}\right)$ is a is a left $D$-module, $\left(M, \varrho_{M}\right)$ is a left $D$-comodule and $\left(r_{M}, r_{M}^{\prime}, s_{M}, s_{M}^{\prime}\right)$ an $(M, D)$-WO compatible with the (co)module structure. Then the simultaneous verification of conditions (yd1-11) and (yd2-11) is equivalent to:

(yd3-11) $\varrho_{M} \circ \varphi_{M}$

$$
=\left(\mu_{D} \otimes M\right) \circ\left(D \otimes r_{M}\right) \circ\left(\left(\left(\mu_{D} \otimes \varphi_{M}\right) \circ\left(D \otimes t_{D, D} \otimes M\right) \circ\left(\delta_{D} \otimes \varrho_{M}\right)\right) \otimes \lambda_{D}\right) \circ\left(D \otimes s_{M}\right) \circ\left(\delta_{D} \otimes M\right) .
$$

Following the same pattern it can be given a characterization of the objects in $\mathcal{Y} \mathcal{D}_{D}^{D}$ via a condition (yd3-rr) analogous to that given in (yd3-1l) for the left-left case:

(yd3-rr) $\rho_{M} \circ \psi_{M}$

$$
=\left(M \otimes \mu_{D}\right) \circ\left(s_{M} \otimes D\right) \circ\left(\lambda_{D} \otimes\left(\left(\psi_{M} \otimes \mu_{D}\right) \circ\left(M \otimes t_{D, D} \otimes D\right) \circ\left(\rho_{M} \otimes \delta_{D}\right)\right)\right) \circ\left(r_{M} \otimes D\right) \circ\left(M \otimes \delta_{D}\right) .
$$

Moreover, when $D$ is such that the antipode $\lambda_{D}$ is invertible, then the corresponding results also hold for ${ }_{D} \mathcal{Y} \mathcal{D}^{D}$ and ${ }^{D} \mathcal{Y} \mathcal{D}_{D}$. Actually, in the left-right case, it can be checked that if $\left(M, \varphi_{M}\right)$ is a left $D$-module, $\left(M, \rho_{M}\right)$ is a right comodule and there exists $\left(r_{M}, r_{M}^{\prime}, s_{M}, s_{M}^{\prime}\right)$ an $(M, D)$-WO compatible with the (co)module structures of $M$, then the simultaneous verification of conditions (yd1-lr) and (yd2-lr) is equivalent to that of

(yd3-lr) $\rho_{M} \circ \varphi_{M}$

$$
=\left(M \otimes\left(\mu_{D} \circ t_{D, D}^{\prime}\right)\right) \circ\left(r_{M}^{\prime} \otimes D\right) \circ\left(\lambda_{D}^{-1} \otimes\left(\left(\varphi_{M} \otimes \mu_{D}\right) \circ\left(D \otimes s_{M} \otimes D\right) \circ\left(\delta_{D} \otimes \rho_{M}\right)\right)\right) \circ\left(\delta_{D} \otimes M\right) .
$$

Analogously, we would deduce the corresponding condition (yd3-rl) for the right-left case:

(yd3-rl) $\varrho_{M} \circ \psi_{M}$

$$
=\left(\left(\mu_{D} \circ t_{D, D}^{\prime}\right) \otimes M\right) \circ\left(D \otimes s_{M}^{\prime}\right) \circ\left(\left(\left(\mu_{D} \otimes \psi_{M}\right) \circ\left(D \otimes r_{M} \otimes D\right) \circ\left(\varrho_{M} \otimes \delta_{D}\right)\right) \otimes \lambda_{D}^{-1}\right) \circ\left(M \otimes \delta_{D}\right) .
$$

Remark 3.10 It is not difficult to see that, if $\left(M, \varphi_{M}, \varrho_{M}\right)$ is an object in ${ }_{D}^{D} \mathcal{Y} \mathcal{D}$, then

$$
\varphi_{M} \circ \nabla_{s_{M}}=\varphi_{M} \circ \nabla_{r_{M}^{\prime}}=\varphi_{M} ; \quad \nabla_{s_{M}} \circ \varrho_{M}=\nabla_{r_{M}^{\prime}} \circ \varrho_{M}=\varrho_{M}
$$

Using condition (yd3-rr) we obtain the analogous equalities corresponding to objects in $\mathcal{Y} \mathcal{D}_{D}^{D}$. Moreover, the result is also verified for objects in ${ }_{D} \mathcal{Y} \mathcal{D}^{D}$ or ${ }^{D} \mathcal{Y} \mathcal{D}_{D}$, although to prove these cases $\lambda_{D}$ is required to be invertible because we need to use (yd3-lr) and (yd3-rl), respectively.

We proceed now to state the main result of this paper.

Theorem 3.11 Let D be a WBHA with invertible antipode. The following statements are equivalent:

(i) $\left(M, \varphi_{M}, \varrho_{M}\right) \in{ }_{D}^{D} \mathcal{Y} \mathcal{D}$,

(ii) $\left(M, \psi_{M}^{\lambda}, \rho_{M}^{\lambda^{-1}}\right) \in \mathcal{Y} \mathcal{D}_{D}^{D}$,

(iii) $\left(M, \psi_{M}^{\lambda^{-1}}, \rho_{M}^{\lambda}\right) \in \mathcal{Y} \mathcal{D}_{D}^{D}$,

(iv) $\left(M, \varphi_{M}, \rho_{M}^{\lambda^{-1}}\right) \in{ }_{D} \mathcal{Y} \mathcal{D}^{D}$,

(v) $\left(M, \psi_{M}^{\lambda^{-1}}, \varrho_{M}\right) \in{ }^{D} \mathcal{Y} \mathcal{D}_{D}$.

Moreover, taking the identity acting on morphisms between Yetter-Drinfeld modules, the above transformations define actually equivalences of categories. 
Proof In virtue of (11), (12), (21) and Remark 3.10, we know that the hypotheses of Propositions 2.13-2.16 remain fulfilled after each transformation on the (co)module structures considered in the statement of the theorem. As a consequence, all the new (co)module structures we generate are advisable in terms of compatibility with the weak operator, so we are reduced to check the respective (yd1)-type and (yd2)-type conditions on each case.

Moreover, in order to prove the equivalence between any two statements it is enough to demonstrate one implication. If this were done, taking the transformations in the (co)module structures suggested by that applied in the direct implication, Propositions 2.13-2.16 guarantee that retracing our steps in the arguments we get the opposite implication. Moreover, if we apply two consecutive transformations the starting structures are recovered. Since this is the situation, it suffices to prove that any statement can be deduced from $(i)$.

For Part (ii), condition (yd1-rr) is checked directly by the following calculations:

$$
\begin{aligned}
& \left(\psi_{M}^{\lambda} \otimes \mu_{D}\right) \circ\left(M \otimes t_{D, D} \otimes D\right) \circ\left(\rho_{M}^{\lambda^{-1}} \otimes\left(\delta_{D} \circ \eta_{D}\right)\right) \\
& =\left(\left(\psi_{M}^{\lambda} \circ\left(M \otimes \Pi_{D}^{R}\right)\right) \otimes D\right) \circ\left(M \otimes \delta_{D}\right) \circ \rho_{M}^{\lambda^{-1}} \\
& =\left(\left(\varphi_{M} \circ r_{M}\right) \otimes D\right) \circ\left(M \otimes\left(\lambda_{D} \circ \Pi_{D}^{R}\right) \otimes D\right) \circ\left(M \otimes\left(\delta_{D} \circ \lambda_{D}^{-1}\right)\right) \circ r_{M}^{\prime} \circ \varrho_{M} \\
& =\left(\left(\varphi_{M} \circ r_{M}\right) \otimes D\right) \circ\left(M \otimes\left(\lambda_{D} \circ \Pi_{D}^{R}\right) \otimes D\right) \circ\left(M \otimes\left(\left(\lambda_{D}^{-1} \otimes \lambda_{D}^{-1}\right) \circ t_{D, D}^{\prime} \circ \delta_{D}\right)\right) \circ r_{M}^{\prime} \circ \varrho_{M} \\
& =\left(\left(\varphi_{M} \circ\left(\Pi_{D}^{L} \otimes M\right)\right) \otimes D\right) \circ\left(\nabla_{r_{M}^{\prime}} \otimes \lambda_{D}^{-1}\right) \circ\left(D \otimes r_{M}^{\prime}\right) \circ\left(t_{D, D}^{\prime} \otimes M\right) \circ\left(\delta_{D} \otimes M\right) \circ \varrho_{M} \\
& =\left(\left(\varphi_{M} \circ\left(\Pi_{D}^{L} \otimes M\right)\right) \otimes \lambda_{D}^{-1}\right) \circ\left(D \otimes r_{M}^{\prime}\right) \circ\left(t_{D, D}^{\prime} \otimes M\right) \circ\left(\delta_{D} \otimes M\right) \circ \varrho_{M} \\
& =\left(M \otimes \lambda_{D}^{-1}\right) \circ r_{M}^{\prime} \circ\left(D \otimes\left(\varphi_{M} \circ\left(\Pi_{D}^{L} \otimes M\right) \circ \varrho_{M}\right)\right) \circ \varrho_{M}=\rho_{M}^{\lambda^{-1}},
\end{aligned}
$$

where the first equality follows because $\left(\Pi_{D}^{R} \otimes D\right) \circ \delta_{D}=\left(D \otimes \mu_{D}\right) \circ\left(t_{D, D} \otimes D\right) \circ\left(D \otimes\left(\delta_{D} \circ \eta_{D}\right)\right)$, the second by definition of the (co)module structure, the third one by anticomultiplicativity of $\lambda_{D}^{-1}$ and on the fourth we use that $\lambda_{D} \circ \Pi_{D}^{R}=\Pi_{D}^{L} \circ \lambda_{D}$, (c4), (c1) and (23). The fifth equality relies on (23) and the equality $\varphi_{M}=\varphi_{M} \circ \nabla_{r_{M}^{\prime}}$; the sixth one relies on compatibility and the seventh is a consequence of the equality $\varphi_{M} \circ\left(\Pi_{D}^{L} \otimes M\right) \circ \varrho_{M}=i d_{M}$ which follows in virtue of (yd1-11).

To prove (yd2-rr), starting from condition (yd2-1l) and composing with $\lambda_{D}$ and $\lambda_{D}^{-1}$ we obtain two equivalent expressions, one for each side of (yd2-11). Specifically, on the right side we have:

$$
\begin{aligned}
&\left(\lambda_{D}{ }^{-1} \otimes M\right) \circ\left(\mu_{D} \otimes M\right) \circ\left(D \otimes r_{M}\right) \circ\left(\left(\varrho_{M} \circ \varphi_{M}\right) \otimes D\right) \circ\left(D \otimes s_{M}\right) \circ\left(\delta_{D} \otimes M\right) \circ\left(\lambda_{D} \otimes M\right) \\
&=\left(\left(\mu_{D} \circ t_{D, D}^{\prime} \circ\left(\lambda_{D}^{-1} \otimes \lambda_{D}^{-1}\right)\right) \otimes M\right) \circ\left(D \otimes r_{M}\right) \circ\left(\left(\varrho_{M} \circ \varphi_{M}\right) \otimes D\right) \circ\left(D \otimes s_{M}\right) \\
& \circ\left(\left(\left(\lambda_{D} \otimes \lambda_{D}\right) \circ t_{D, D} \circ \delta_{D}\right) \otimes M\right) \\
&=\left(\left(\mu_{D} \circ t_{D, D}^{\prime} \circ\left(\lambda_{D}^{-1} \otimes D\right)\right) \otimes M\right) \circ\left(D \otimes r_{M}\right) \circ\left(\left(\nabla_{r_{M}^{\prime}} \circ \varrho_{M} \circ \varphi_{M} \circ \nabla_{r_{M}^{\prime}}\right) \otimes D\right) \circ\left(D \otimes s_{M}\right) \\
& \circ\left(\left(\left(\lambda_{D} \otimes D\right) \circ t_{D, D} \circ \delta_{D}\right) \otimes M\right) \\
&=\left(\left(\mu_{D} \circ t_{D, D}^{\prime}\right) \otimes M\right) \circ\left(D \otimes r_{M}\right) \circ\left(r_{M} \otimes D\right) \circ\left(\left(\rho_{M}^{\lambda^{-1}} \circ \psi_{M}^{\lambda}\right) \otimes D\right) \circ\left(r_{M}^{\prime} \otimes D\right) \circ\left(D \otimes s_{M}\right) \\
& \circ\left(\left(t_{D, D} \circ \delta_{D}\right) \otimes M\right) \\
&= r_{M} \circ\left(M \otimes\left(\mu_{D} \circ t_{D, D}^{\prime}\right)\right) \circ\left(\left(\rho_{M}^{\lambda^{-1}} \circ \psi_{M}^{\lambda}\right) \otimes D\right) \circ\left(M \otimes t_{D, D}\right) \circ\left(s_{M} \otimes D\right) \circ\left(D \otimes r_{M}^{\prime}\right) \circ\left(\delta_{D} \otimes M\right) .
\end{aligned}
$$

In the above calculations, on the first equality we apply the properties of the antipode $\lambda_{D}$ and its inverse $\lambda_{D}^{-1}$, the second follows by (21) and Remark 3.10, the third one by (21) and the definitions of $\rho_{M}^{\lambda^{-1}}$ and $\psi_{M}^{\lambda}$, and on the last one we use conditions (c2-1), (c1) and (c4-1).

For the left side, by the same arguments we obtain that:

$$
\begin{aligned}
\left(\lambda_{D}^{-1} \otimes M\right) \circ\left(\left(\mu_{D} \otimes \varphi_{M}\right) \circ\left(D \otimes t_{D, D} \otimes M\right) \circ\left(\delta_{D} \otimes \varrho_{M}\right)\right) \circ\left(\lambda_{D} \otimes M\right) \\
=\left(\left(\mu_{D} \circ t_{D, D}^{\prime} \circ\left(\lambda_{D}^{-1} \otimes \lambda_{D}^{-1}\right)\right) \otimes \varphi_{M}\right) \circ\left(D \otimes t_{D, D} \otimes M\right) \circ\left(\left(\left(\lambda_{D} \otimes \lambda_{D}\right) \circ t_{D, D} \circ \delta_{D}\right) \otimes \varrho_{M}\right) \\
=\left(\left(\mu_{D} \circ t_{D, D}^{\prime} \circ\left(D \otimes \lambda_{D}^{-1}\right)\right) \otimes\left(\varphi_{M} \circ \nabla_{r_{M}^{\prime}}\right) \circ\left(D \otimes t_{D, D} \otimes M\right)\right. \\
\quad \circ\left(\left(\left(D \otimes \lambda_{D}\right) \circ t_{D, D} \circ \delta_{D}\right) \otimes\left(\nabla_{r_{M}^{\prime}} \circ \varrho_{M}\right)\right) \\
=\left(\left(\mu_{D} \circ t_{D, D}^{\prime}\right) \otimes\left(\psi_{M}^{\lambda} \circ r_{M}^{\prime}\right)\right) \circ\left(D \otimes t_{D, D} \otimes M\right) \circ\left(\left(t_{D, D} \circ \delta_{D}\right) \otimes\left(r_{M} \circ \rho_{M}^{\lambda^{-1}}\right)\right) .
\end{aligned}
$$


The strategy of the proof consists of demonstrating that each side of the equality occurring on (yd2-rr) can be obtained by composing with $r_{M}$ and $r_{M}^{\prime}$ the arrows appearing at the end of the two precedent blocks of equalities, as we already know that these are equivalent. With this purpose, starting with the end of the first block we get that:

$$
\begin{aligned}
r_{M}^{\prime} \circ & r_{M} \circ\left(M \otimes\left(\mu_{D} \circ t_{D, D}^{\prime}\right)\right) \circ\left(\left(\rho_{M}^{\lambda^{-1}} \circ \psi_{M}^{\lambda}\right) \otimes D\right) \circ\left(M \otimes t_{D, D}\right) \circ\left(s_{M} \otimes D\right) \circ\left(D \otimes r_{M}^{\prime}\right) \\
& \circ\left(\delta_{D} \otimes M\right) \circ r_{M} \\
= & \nabla_{r_{M}} \circ\left(M \otimes\left(\mu_{D} \circ t_{D, D}^{\prime}\right)\right) \circ\left(\left(\rho_{M}^{\lambda^{-1}} \circ \psi_{M}^{\lambda}\right) \otimes D\right) \circ\left(M \otimes t_{D, D}\right) \circ\left(s_{M} \otimes D\right) \circ\left(D \otimes \nabla_{r_{M}}\right) \circ\left(r_{M} \otimes D\right) \\
& \circ\left(M \otimes \delta_{D}\right) \\
= & \nabla_{r_{M}} \circ\left(M \otimes\left(\mu_{D} \circ t_{D, D}^{\prime}\right)\right) \circ\left(\rho_{M}^{\lambda^{-1}} \otimes D\right) \circ s_{M} \circ\left(D \otimes\left(\psi_{M}^{\lambda} \circ \nabla_{r_{M}}\right)\right) \circ\left(r_{M} \otimes D\right) \circ\left(M \otimes \delta_{D}\right) \\
= & \left(M \otimes\left(\mu_{D} \circ t_{D, D}^{\prime}\right)\right) \circ\left(\left(\nabla_{r_{M}} \circ \rho_{M}^{\lambda^{-1}}\right) \otimes D\right) \circ s_{M} \circ\left(D \otimes \psi_{M}^{\lambda}\right) \circ\left(r_{M} \otimes D\right) \circ\left(M \otimes \delta_{D}\right) \\
= & \left(M \otimes\left(\mu_{D} \circ \nabla_{D, D}\right)\right) \circ\left(s_{M} \otimes D\right) \circ\left(D \otimes\left(\rho_{M}^{\lambda-1} \circ \psi_{M}^{\lambda}\right)\right) \circ\left(r_{M} \otimes D\right) \circ\left(M \otimes \delta_{D}\right) \\
= & \left(M \otimes \mu_{D}\right) \circ\left(s_{M} \otimes D\right) \circ\left(D \otimes\left(\rho_{M}^{\lambda-1} \circ \psi_{M}^{\lambda}\right)\right) \circ\left(r_{M} \otimes D\right) \circ\left(M \otimes \delta_{D}\right) .
\end{aligned}
$$

In the above calculations, the first equality follows by (c4) and the definition of $\nabla_{r_{M}}$ and the second one by compatibility of $\psi_{M}^{\lambda}$. In the third one we use Remark 3.10 and the equality

$$
\nabla_{r_{M}} \circ\left(M \otimes\left(\mu_{D} \circ t_{D, D}^{\prime}\right)\right)=\left(M \otimes\left(\mu_{D} \circ t_{D, D}^{\prime}\right)\right) \circ\left(\nabla_{r_{M}} \otimes D\right),
$$

which is a direct consequence of (25). On the fourth equality we use Remark 3.10 and compatibility of $\rho_{M}^{\lambda^{-1}}$, and the last one follows by (b1-1).

Applying the same composition to the end of the second block, in virtue of (c4), (c1), the definition of (M,D)-WO, (13), compatibilities of $\rho_{M}^{\lambda^{-1}}$ and $\psi_{M}^{\lambda}$, the equality

$$
\left(\nabla_{r_{M}} \otimes D\right) \circ\left(M \otimes\left(t_{D, D} \circ \delta_{D}\right)\right)=\left(M \otimes\left(t_{D, D} \circ \delta_{D}\right)\right) \circ \nabla_{r_{M}},
$$

which follows by (26), as well as Remark 3.10, we obtain:

$$
\begin{aligned}
& r_{M}^{\prime} \circ\left(\left(\mu_{D} \circ t_{D, D}^{\prime}\right) \otimes\left(\psi_{M}^{\lambda} \circ r_{M}^{\prime}\right)\right) \circ\left(D \otimes t_{D, D} \otimes M\right) \circ\left(\left(t_{D, D} \circ \delta_{D}\right) \otimes\left(r_{M} \circ \rho_{M}^{\lambda^{-1}}\right)\right) \circ r_{M} \\
& =\left(M \otimes\left(\mu_{D} \circ t_{D, D}^{\prime}\right)\right) \circ\left(r_{M}^{\prime} \otimes D\right) \circ\left(D \otimes r_{M}^{\prime}\right) \circ\left(D \otimes D \otimes\left(\psi_{M}^{\lambda} \circ r_{M}^{\prime}\right)\right) \circ\left(D \otimes t_{D, D} \otimes M\right) \\
& \circ\left(D \otimes D \otimes\left(r_{M} \circ \rho_{M}^{\lambda^{-1}}\right)\right) \circ\left(D \otimes r_{M}\right) \circ\left(r_{M} \otimes D\right) \circ\left(M \otimes\left(t_{D, D} \circ \delta_{D}\right)\right) \\
& =\left(\psi_{M}^{\lambda} \otimes\left(\mu_{D} \circ t_{D, D}^{\prime}\right)\right) \circ\left(M \otimes t_{D, D}^{\prime} \otimes D\right) \circ\left(r_{M}^{\prime} \otimes t_{D, D}^{\prime}\right) \circ\left(D \otimes r_{M}^{\prime} \otimes D\right) \circ\left(D \otimes D \otimes r_{M}^{\prime}\right) \\
& \circ\left(D \otimes t_{D, D} \otimes M\right) \circ\left(D \otimes D \otimes r_{M}\right) \circ\left(D \otimes r_{M} \otimes D\right) \circ\left(r_{M} \otimes t_{D, D}\right) \circ\left(M \otimes t_{D, D} \otimes D\right) \\
& \circ\left(\rho_{M}^{\lambda^{-1}} \otimes\left(t_{D, D} \circ \delta_{D}\right)\right) \\
& =\left(\psi_{M}^{\lambda} \otimes\left(\mu_{D} \circ t_{D, D}^{\prime}\right)\right) \circ\left(M \otimes t_{D, D}^{\prime} \otimes D\right) \circ\left(r_{M}^{\prime} \otimes \nabla_{D, D}\right) \circ\left(D \otimes r_{M}^{\prime} \otimes D\right) \circ\left(D \otimes D \otimes \nabla_{r_{M}}\right) \\
& \circ\left(D \otimes r_{M} \otimes D\right) \circ\left(r_{M} \otimes t_{D, D}\right) \circ\left(M \otimes t_{D, D} \otimes D\right) \circ\left(\rho_{M}^{\lambda^{-1}} \otimes\left(t_{D, D} \circ \delta_{D}\right)\right) \\
& =\left(\psi_{M}^{\lambda} \otimes\left(\mu_{D} \circ t_{D, D}^{\prime}\right)\right) \circ\left(M \otimes t_{D, D}^{\prime} \otimes D\right) \circ\left(r_{M}^{\prime} \otimes D \otimes D\right) \circ\left(D \otimes \nabla_{r_{M}} \otimes D\right) \circ\left(r_{M} \otimes t_{D, D}\right) \\
& \circ\left(M \otimes t_{D, D} \otimes D\right) \circ\left(\left(\nabla_{r_{M}} \circ \rho_{M}^{\lambda^{-1}}\right) \otimes\left(t_{D, D} \circ \delta_{D}\right)\right) \\
& =\left(\left(\psi_{M}^{\lambda} \circ \nabla_{r_{M}}\right) \otimes\left(\mu_{D} \circ t_{D, D}^{\prime}\right)\right) \circ\left(M \otimes t_{D, D}^{\prime} \otimes D\right) \circ\left(\nabla_{r_{M}} \otimes t_{D, D}\right) \circ\left(M \otimes t_{D, D} \otimes D\right) \\
& \circ\left(\rho_{M}^{\lambda^{-1}} \otimes\left(t_{D, D} \circ \delta_{D}\right)\right) \\
& =\left(\psi_{M}^{\lambda} \otimes\left(\mu_{D} \circ t_{D, D}^{\prime}\right)\right) \circ\left(M \otimes t_{D, D}^{\prime} \otimes D\right) \circ\left(\nabla_{r_{M}} \otimes D \otimes D\right) \circ\left(M \otimes\left(t_{D, D} \circ \delta_{D}\right) \otimes D\right) \\
& \circ\left(M \otimes t_{D, D}\right) \circ\left(\rho_{M}^{\lambda^{-1}} \otimes D\right) \\
& =\left(\psi_{M}^{\lambda} \otimes\left(\mu_{D} \circ t_{D, D}^{\prime}\right)\right) \circ\left(M \otimes\left(\nabla_{D, D} \circ \delta_{D}\right) \otimes D\right) \circ\left(\nabla_{r_{M}} \otimes D\right) \circ\left(M \otimes t_{D, D}\right) \circ\left(\rho_{M}^{\lambda^{-1}} \otimes D\right) \\
& =\left(\left(\psi_{M}^{\lambda} \circ \nabla_{r_{M}}\right) \otimes\left(\mu_{D} \circ \nabla_{D, D}\right)\right) \circ\left(M \otimes t_{D, D} \otimes D\right) \circ\left(\rho_{M}^{\lambda^{-1}} \otimes \delta_{D}\right) \\
& =\left(\psi_{M}^{\lambda} \otimes \mu_{D}\right) \circ\left(M \otimes t_{D, D} \otimes D\right) \circ\left(\rho_{M}^{\lambda^{-1}} \otimes \delta_{D}\right) .
\end{aligned}
$$


In the foregoing computations, the first equality follows by (c4) and (c1); in the second one we use the compatibility of $\rho_{M}^{\lambda^{-1}}$ and $\psi_{M}^{\lambda}$; the third relies on (c1) and (2); the fourth follows by the equality $\left(D \otimes \nabla_{r_{M}}\right) \circ$ $\left(r_{M} \otimes D\right) \circ\left(M \otimes t_{D, D}\right)=\left(r_{M} \otimes D\right) \circ\left(M \otimes t_{D, D}\right) \circ\left(\nabla_{r_{M}} \otimes D\right)$; the fifth because $\nabla_{r_{M}} \circ \rho_{M}^{\lambda^{-1}}=\rho_{M}^{\lambda^{-1}}$ and $\psi_{M}^{\lambda} \circ \nabla_{r_{M}}=\psi_{M}^{\lambda}$; the sixth one is a consequence of (a1) and (b3-3); in the the seventh we apply Proposition 2.10; the eighth one follows by (b2-1), (b3-3) and (2); finally, the last equality is a consequence of (b1-1) and the equality $\psi_{M}^{\lambda} \circ \nabla_{r_{M}}=\psi_{M}^{\lambda}$.

To demonstrate that $(i)$ implies $($ iii $)$ we can follow a similar pattern.

In order to prove that $(i)$ implies $(i v)$, we begin by checking condition (yd2-lr):

$$
\begin{aligned}
(M \otimes & \left.\left(\mu_{D} \circ t_{D, D}^{\prime}\right)\right) \circ\left(r_{M}^{\prime} \otimes D\right) \circ\left(D \otimes\left(\rho_{M}^{\lambda^{-1}} \circ \varphi_{M}\right)\right) \circ\left(\delta_{D} \otimes M\right) \\
= & \left(M \otimes\left(\mu_{D} \circ t_{D, D}^{\prime}\right)\right) \circ\left(r_{M}^{\prime} \otimes \lambda_{D}^{-1}\right) \circ\left(D \otimes r_{M}^{\prime}\right) \circ\left(D \otimes\left(\varrho_{M} \circ \varphi_{M}\right)\right) \circ\left(\delta_{D} \otimes M\right) \\
= & r_{M}^{\prime} \circ\left(\left(\mu_{D} \circ t_{D, D}^{\prime}\right) \otimes M\right) \circ\left(D \otimes \lambda_{D}^{-1} \otimes M\right) \circ\left(D \otimes\left(\varrho_{M} \circ \varphi_{M}\right)\right) \circ\left(\delta_{D} \otimes M\right) \\
= & r_{M}^{\prime} \circ\left(\left(\mu_{D} \circ t_{D, D}^{\prime}\right) \otimes M\right) \circ\left(\left(\lambda_{D}^{-1} \circ \lambda_{D}\right) \otimes\left(\lambda_{D}^{-1} \circ \mu_{D}\right) \otimes M\right) \circ\left(D \otimes \mu_{D} \otimes r_{M}\right) \\
& \circ\left(D \otimes D \otimes D \otimes \varphi_{M} \otimes \lambda_{D}\right) \circ\left(D \otimes D \otimes t_{D, D} \otimes M \otimes D\right) \circ\left(D \otimes \delta_{D} \otimes \varrho_{M} \otimes D\right) \\
& \circ\left(\delta_{D} \otimes s_{M}\right) \circ\left(\delta_{D} \otimes M\right) \\
= & r_{M}^{\prime} \circ\left(\left(\lambda_{D}^{-1} \circ \mu_{D}\right) \otimes M\right) \circ\left(\mu_{D} \otimes r_{M}\right) \circ\left(D \otimes D \otimes \varphi_{M} \otimes \lambda_{D}\right) \circ\left(\Pi_{D}^{R} \otimes t_{D, D} \otimes M \otimes D\right) \\
& \circ\left(\delta_{D} \otimes \varrho_{M} \otimes D\right) \circ\left(D \otimes s_{M}\right) \circ\left(\delta_{D} \otimes M\right) \\
= & r_{M}^{\prime} \circ\left(\left(\lambda_{D}^{-1} \circ \mu_{D}\right) \otimes M\right) \circ\left(D \otimes r_{M}\right) \circ\left(D \otimes \varphi_{M} \otimes \lambda_{D}\right) \circ\left(t_{D, D} \otimes M \otimes D\right) \circ\left(D \otimes \varrho_{M} \otimes D\right) \\
& \circ\left(D \otimes s_{M}\right) \circ\left(\delta_{D} \otimes M\right) \\
= & \left(M \otimes\left(\lambda_{D}^{-1} \circ \mu_{D}\right)\right) \circ\left(r_{M}^{\prime} \otimes D\right) \circ\left(D \otimes \nabla_{r_{M}}\right) \circ\left(D \otimes \varphi_{M} \otimes \lambda_{D}\right) \circ\left(t_{D, D} \otimes M \otimes D\right) \\
& \circ\left(D \otimes \varrho_{M} \otimes D\right) \circ\left(D \otimes s_{M}\right) \circ\left(\delta_{D} \otimes M\right) \\
= & \left(M \otimes\left(\lambda_{D}^{-1} \circ \mu_{D}\right)\right) \circ\left(r_{M}^{\prime} \otimes \mu_{D}\right) \circ\left(D \otimes r_{M}^{\prime} \otimes D\right) \circ\left(D \otimes \eta_{D} \otimes \varphi_{M} \otimes \lambda_{D}\right) \circ\left(t_{D, D} \otimes M \otimes D\right) \\
& \circ\left(D \otimes \varrho_{M} \otimes D\right) \circ\left(D \otimes s_{M}\right) \circ\left(\delta_{D} \otimes M\right) \\
= & \left(M \otimes\left(\lambda_{D}^{-1} \circ \mu_{D}\right)\right) \circ\left(r_{M}^{\prime} \otimes D\right) \circ\left(D \otimes \varphi_{M} \otimes \lambda_{D}\right) \circ\left(t_{D, D} \otimes M \otimes D\right) \circ\left(D \otimes \varrho_{M} \otimes D\right) \\
& \circ\left(D \otimes s_{M}\right) \circ\left(\delta_{D} \otimes M\right) \\
= & \left(\varphi_{M} \otimes\left(\lambda_{D}^{-1} \circ \mu_{D}\right)\right) \circ\left(D \otimes r_{M}^{\prime} \otimes \lambda_{D}\right) \circ\left(\nabla_{D, D} \otimes s_{M}\right) \circ\left(D \otimes t_{D, D} \otimes M\right) \circ\left(\delta_{D} \otimes \varrho_{M}\right) \\
= & \left(\varphi_{M} \otimes\left(\mu_{D} \circ t_{D, D}^{\prime} \circ\left(\lambda_{D}^{-1} \otimes \lambda_{D}^{-1}\right)\right)\right) \circ\left(D \otimes r_{M}^{\prime} \otimes \lambda_{D}\right) \circ\left(D \otimes D \otimes s_{M}\right) \circ\left(D \otimes t_{D, D} \otimes M\right) \circ\left(\delta_{D} \otimes \varrho_{M}\right) \\
= & \left(\varphi_{M} \otimes\left(\mu_{D} \circ \nabla_{D, D}\right)\right) \circ\left(D \otimes s_{M} \otimes D\right) \circ\left(\delta_{D} \otimes \rho_{M}^{\lambda^{-1}}\right) \\
= & \left(\varphi_{M} \otimes \mu_{D}\right) \circ\left(D \otimes s_{M} \otimes D\right) \circ\left(\delta_{D} \otimes \rho_{M}^{\lambda^{-1}}\right) .
\end{aligned}
$$

In the above calculations, the first equality follows by the definition of $\rho_{M}^{\lambda^{-1}}$; the second one by (c1), (c4-2) and (21); in the third we apply (yd3-11); the fourth one relies on the antimultiplicativity of $\lambda_{D}^{-1}$; the fifth is a consequence of the equality

$$
\left(\mu_{D} \otimes \varphi_{M}\right) \circ\left(\Pi_{D}^{R} \otimes t_{D, D} \otimes M\right) \circ\left(\delta_{D} \otimes \varrho_{M}\right)=\left(D \otimes \varphi_{M}\right) \circ\left(t_{D, D} \otimes M\right) \circ\left(D \otimes \varrho_{M}\right) ;
$$

the sixth follows by (21) and (c4-2); in the seventh we apply (c3-1); the eighth uses (c4-2); the ninth (2) and compatibility of the (co)module structure; the tenth (a2-2), (b2-1) and the antimultiplicativity of $\lambda_{D}^{-1}$; the eleventh one follows by (c2-1), (2) and (9); finally, the last one is a consequence of (b1-1).

Finally we verify condition (yd1-lr):

$$
\begin{aligned}
\left(\varphi_{M} \otimes \mu_{D}\right) \circ\left(D \otimes s_{M} \otimes D\right) \circ\left(\left(\delta_{D} \circ \eta_{D}\right) \otimes \rho_{M}^{\lambda^{-1}}\right) \\
=\left(M \otimes\left(\mu_{D} \circ t_{D, D}^{\prime}\right)\right) \circ\left(r_{M}^{\prime} \otimes D\right) \circ\left(D \otimes r_{M}^{\prime}\right) \circ\left(D \otimes \lambda_{D}^{-1} \otimes M\right) \circ\left(D \otimes \varrho_{M}\right) \circ\left(D \otimes \varphi_{M}\right) \\
\quad \circ\left(\left(\delta_{D} \circ \eta_{D}\right) \otimes M\right) \\
=\left(M \otimes\left(\mu_{D} \circ t_{D, D}^{\prime}\right)\right) \circ\left(r_{M}^{\prime} \otimes D\right) \circ\left(D \otimes r_{M}^{\prime}\right) \circ\left(D \otimes \lambda_{D}^{-1} \otimes M\right) \circ\left(D \otimes \varrho_{M}\right) \circ\left(\bar{\Pi}_{D}^{L} \otimes M\right) \circ \varrho_{M} \\
=r_{M}^{\prime} \circ\left(\left(\mu_{D} \circ t_{D, D}^{\prime} \circ\left(\bar{\Pi}_{D}^{L} \otimes \lambda_{D}^{-1}\right) \circ \delta_{D}\right) \otimes M\right) \circ \varrho_{M}=\rho_{M}^{\lambda^{-1}} .
\end{aligned}
$$


In the preceding equalities, the first one follows by the definition of $\rho_{M}^{\lambda^{-1}}$; the second because

$$
\left(D \otimes \varphi_{M}\right) \circ\left(\left(\delta_{D} \circ \eta_{D}\right) \otimes M\right)=\left(\bar{\Pi}_{D}^{L} \otimes M\right) \circ \varrho_{M},
$$

that in turns relies on the equality $\left(\Pi_{D}^{L} \otimes M\right) \circ \varrho_{M} \circ \varphi_{M}=\left(\Pi_{D}^{L} \otimes \varphi_{M}\right) \circ\left(\delta_{D} \otimes M\right)$; in the third one we use (c1) and (c4-2); finally, the last one is true because $\mu_{D} \circ t_{D, D}^{\prime} \circ\left(\bar{\Pi}_{D}^{L} \otimes \lambda_{D}^{-1}\right) \circ \delta_{D}=\lambda_{D}^{-1}$.

At this point, instead of demonstrating that $(i)$ implies $(v)$, we infer $(v)$ from $(i v)$ because this implication follows from if $\left(M, \varphi_{M}, \rho_{M}\right) \in{ }_{D} \mathcal{Y} \mathcal{D}^{D}$ then $\left(M, \psi_{M}^{\lambda^{-1}}, \rho_{M}^{\lambda}\right) \in{ }^{D} \mathcal{Y} \mathcal{D}_{D}$. The proof is similar to that which establishes (i) implies (ii).

To finish the proof of the theorem, note that the definition of the morphisms in the Yetter-Drinfeld categories and the fact that the transformations taken into account act as the identity on morphisms ensure that we actually have equivalences of categories.

In the above theorem we have seen the categorical equivalences between the various categories of Yetter-Drinfeld modules obtained by changing the side of the (co)module structure. The natural question is if it is possible to obtain similar categorical equivalences by replacing the WBHA $D$ for $D^{o p}, D^{\text {coop }}, D^{o p^{c o o p}}$ or $D^{c o o p}{ }^{o p}$, respectively. In the following result we establish this equivalences. Note that our case is slightly different to the one developed in [15], because in our setting $D^{\text {op coop }}$ and $D^{\text {coop }}$ do not coincide.

Theorem 3.12 Let D be a WBHA with invertible antipode. The following statements are equivalent:

(i) $\left(M, \varphi_{M}, \varrho_{M}\right) \in_{D}^{D} \mathcal{Y} \mathcal{D}$,

(ii) $\left(M, \psi_{M}, \rho_{M}^{\prime}\right) \in \mathcal{Y} \mathcal{D}_{D^{\text {coop pop }}}^{D^{\text {coop }} \text {, }}$

(iii) $\left(M, \psi_{M}^{\prime}, \rho_{M}\right) \in \mathcal{Y} \mathcal{D}_{D^{\mathrm{op}}{ }^{\mathrm{coop}} \text {, }}^{D^{\mathrm{op}}}$

(iv) $\left(M, \varphi_{M}, \rho_{M}^{\prime}\right) \in \in_{D^{\text {coop }}} \mathcal{Y} \mathcal{D}^{D^{\text {coop }}}$,

(v) $\left(M, \psi_{M}^{\prime}, \varrho_{M}\right) \in \in^{D^{\text {op }}} \mathcal{Y} \mathcal{D}_{D^{\text {op }}}$.

Moreover, taking the identity acting on morphisms between Yetter-Drinfeld modules, the above transformations define actually equivalences of categories.

Proof Firstly, note that if we take $\left(r_{M}, r_{M}^{\prime}, s_{M}, s_{M}^{\prime}\right)$ as the compatible $(M, D)$-WO on statement $(i)$, then we will take $\left(s_{M}^{\prime}, s_{M}, r_{M}^{\prime}, r_{M}\right)$ on (iv) and $(v)$ following the results of Propositions 2.13 and 2.15. However, it is relevant to point out that as the roles of the morphisms $r_{M}$ and $s_{M}^{\prime}$, respectively $r_{M}^{\prime}$ and $s_{M}$, are symmetric, so in virtue of Propositions 2.13-2.16 it is completely irrelevant the particular choice of the $(M, D)$-WO done on the starting statement; the indicated transformations will led to a compatible weak operator.

Let $\left(M, \varphi_{M}, \varrho_{M}\right)$ be in ${ }_{D}^{D} \mathcal{Y} \mathcal{D}$. Then, by Theorem 3.11, it holds that $\left(M, \psi_{M}^{\lambda}, \rho_{M}^{\lambda^{-1}}\right)$ is in $\mathcal{Y} \mathcal{D}_{D}^{D}$. Keeping this fact in mind, and having into account the definitions of $\psi_{M}^{\lambda}$ and $\rho_{M}^{\lambda^{-1}}$, by condition (yd1-rr) it results that:

$$
\begin{aligned}
\rho_{M}^{\prime} & =\left(M \otimes \lambda_{D}\right) \circ \rho_{M}^{\lambda^{-1}} \\
& =\left(\psi_{M}^{\lambda} \otimes\left(\lambda_{D} \circ \mu_{D}\right)\right) \circ\left(M \otimes t_{D, D} \otimes D\right) \circ\left(\rho_{M}^{\lambda^{-1}} \otimes\left(\delta_{D} \circ \eta_{D}\right)\right) \\
& =\left(\psi_{M} \otimes\left(\mu_{D} \circ t_{D, D}\right)\right) \circ\left(M \otimes t_{D, D} \otimes D\right) \circ\left(\rho_{M}^{\prime} \otimes\left(\left(\lambda_{D} \otimes \lambda_{D}\right) \circ \delta_{D} \circ \eta_{D}\right)\right) \\
& =\left(\psi_{M} \otimes \mu_{D^{\text {coop }}}\right) \circ\left(M \otimes t_{D, D} \otimes D\right) \circ\left(\rho_{M}^{\prime} \otimes\left(\delta_{D^{\text {coop }}} \circ \eta_{D^{\text {coop }}}\right)\right) .
\end{aligned}
$$

In the above computations, the first equality follows by the definition of $\rho_{M}^{\lambda^{-1}}$; the second one by (yd1-rr) and the third and fourth ones by the anti(co)multiplicativity of $\lambda_{D}$.

Composing with the antipode and its inverse in condition (yd2-rr) for $\left(M, \psi_{M}^{\lambda}, \rho_{M}^{\lambda^{-1}}\right)$ and using (21) we deduce similarly that condition (yd2-rr) for $\left(M, \psi_{M}, \rho_{M}^{\prime}\right)$ as required in (ii) is verified. As a consequence we obtain that (i) implies (ii). In a similar way, using that $\left(M, \psi_{M}^{\lambda^{-1}}, \rho_{M}^{\lambda}\right)$ is in $\mathcal{Y} \mathcal{D}_{D}^{D}$ we can prove that $(i)$ implies (iii).

To prove that (i) implies (iv), condition (yd1-lr) referred to $\left(M, \varphi_{M}, \rho_{M}^{\prime}\right)$ with respect to the $(M, D)$-WO $\left(s_{M}^{\prime}, s_{M}, r_{M}^{\prime}, r_{M}\right)$ can be proved using condition (yd1-1l) with respect to the $(M, D)$-WO $\left(r_{M}, r_{M}^{\prime}, s_{M}, s_{M}^{\prime}\right)$, and the definitions of WBHA and weak Yang-Baxter operator as follows: 


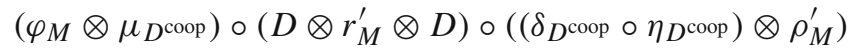

$$
\begin{aligned}
& =\left(\varphi_{M} \otimes \mu_{D}\right) \circ\left(D \otimes r_{M}^{\prime} \otimes D\right) \circ\left(\left(t_{D, D}^{\prime} \circ \delta_{D} \circ \eta_{D}\right) \otimes\left(r_{M}^{\prime} \circ \varrho_{M}\right)\right) \\
& =\left(\varphi_{M} \otimes D\right) \circ\left(D \otimes r_{M}^{\prime}\right) \circ\left(D \otimes \mu_{D} \otimes M\right) \circ\left(\left(t_{D, D}^{\prime} \circ \delta_{D} \circ \eta_{D}\right) \otimes \varrho_{M}\right) \\
& =\left(\varphi_{M} \otimes D\right) \circ\left(D \otimes r_{M}^{\prime}\right) \circ\left(\left(t_{D, D}^{\prime} \circ t_{D, D}\right) \otimes M\right) \circ\left(D \otimes \mu_{D} \otimes M\right) \circ\left(\left(t_{D, D}^{\prime} \circ \delta_{D} \circ \eta_{D}\right) \otimes \varrho_{M}\right) \\
& =r_{M}^{\prime} \circ\left(D \otimes \varphi_{M}\right) \circ\left(t_{D, D} \otimes M\right) \circ\left(D \otimes \mu_{D} \otimes M\right) \circ\left(\left(t_{D, D}^{\prime} \circ \delta_{D} \circ \eta_{D}\right) \otimes \varrho_{M}\right) \\
& =r_{M}^{\prime} \circ\left(\mu_{D} \otimes \varphi_{M}\right) \circ\left(D \otimes t_{D, D} \otimes M\right) \circ\left(\left(\nabla_{D, D} \circ \delta_{D} \circ \eta_{D}\right) \otimes \varrho_{M}\right) \\
& =r_{M}^{\prime} \circ \varrho_{M} \\
& =\rho_{M}^{\prime} \text {. }
\end{aligned}
$$

In the foregoing calculations, the first and the last equalities use the definition of $\rho_{M}^{\prime}$; the second one (c4-2); the third one (2), (b1-3) and (a3-2); the fourth follows by compatibility for the module structure; the fifth relies on (b3-2); finally the sixth one is a consequence of (2), (b2-1) and (yd1-11).

Following the same ideas and taking into account that $t_{D^{\text {coop }}, D^{\text {coop }}}^{\prime}=t_{D, D}$, we get (yd2-lr). Indeed:

$$
\begin{aligned}
& \left(\varphi_{M} \otimes \mu_{D^{\text {coop }}}\right) \circ\left(D \otimes r_{M}^{\prime} \otimes D\right) \circ\left(\delta_{D^{\text {coop }}} \otimes \rho_{M}^{\prime}\right) \\
& =\left(\varphi_{M} \otimes D\right) \circ\left(D \otimes r_{M}^{\prime}\right) \circ\left(D \otimes \mu_{D} \otimes M\right) \circ\left(t_{D, D}^{\prime} \otimes D \otimes M\right) \circ\left(D \otimes\left(t_{D, D}^{\prime} \circ t_{D, D}\right) \otimes M\right) \circ\left(\delta_{D} \otimes \varrho_{M}\right) \\
& =r_{M}^{\prime} \circ\left(\mu_{D} \otimes \varphi_{M}\right) \circ\left(D \otimes t_{D, D} \otimes M\right) \circ\left(\delta_{D} \otimes \varrho_{M}\right) \\
& =r_{M}^{\prime} \circ\left(\mu_{D} \otimes M\right) \circ\left(D \otimes r_{M}\right) \circ\left(\left(\varrho_{M} \circ \varphi_{M}\right) \otimes D\right) \circ\left(D \otimes s_{M}\right) \circ\left(\delta_{D} \otimes M\right) \\
& =\left(M \otimes \mu_{D}\right) \circ\left(r_{M}^{\prime} \otimes D\right) \circ\left(D \otimes \nabla_{r_{M}}\right) \circ\left(\varrho_{M} \otimes D\right) \circ s_{M} \circ\left(D \otimes \varphi_{M}\right) \circ\left(\left(t_{D, D}^{\prime} \circ \delta_{D}\right) \otimes M\right)
\end{aligned}
$$

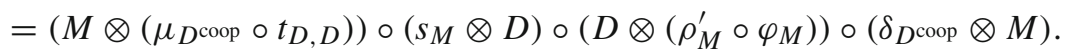

In the above computations the first equality follows by (c4-2) and the properties of the weak Yang-Baxter operator; the second one by compatibility for the module structure; in the third equality we apply (yd2-11); the fourth uses (c4-2) and compatibility for the module structure; finally the last one is a consequence of the properties of the morphism $\nabla_{r_{M}}$.

Finally, to prove that (i) implies (v) we can follow the same pattern.

Example 3.13 We will finish this work with an example closely connected with the notion of WBHA-projection. We briefly recall the definition and main properties of such construction. The details can be found in Section 1 of [3].

Given a WBHA $D$, a WBHA-projection over $D$ is a triple $(B, f, g)$ where $B$ is a WBHA, and $f: D \rightarrow B$, $g: B \rightarrow D$ are morphisms of WBHAs such that $g \circ f=i d_{D}$ and satisfy the following equalities:

(a) $(B \otimes(f \circ g)) \circ t_{B, B}=t_{B, B} \circ((f \circ g) \otimes B)$,

(b) $((f \circ g) \otimes B) \circ t_{B, B}=t_{B, B} \circ(B \otimes(f \circ g))$.

A morphism between two WBHA-projections $(B, f, g)$ and $\left(B^{\prime}, f^{\prime}, g^{\prime}\right)$ associated to $D$ is a morphism of WBHA $h: B \rightarrow B^{\prime}$ such that $h \circ f=f^{\prime}$ and $g^{\prime} \circ h=g$.

Given $(B, f, g)$ a WBHA-projection over $D$ we define the object $B_{D}$ as the image of the idempotent morphism

$$
q_{D}^{B}:=i d_{B} *\left(f \circ \lambda_{D} \circ g\right)
$$

and denote by $i_{D}^{B}$ and $p_{D}^{B}$ the morphisms such that $p_{D}^{B} \circ i_{D}^{B}=i d_{B_{D}}$ and $i_{D}^{B} \circ p_{D}^{B}=q_{D}^{B}$.

It holds that the triple $\left(B_{D}, \varphi_{B_{D}}:=p_{D}^{B} \circ \mu_{B} \circ\left(f \otimes i_{D}^{B}\right), \varrho_{B_{D}}=\left(g \otimes p_{D}^{B}\right) \circ \delta_{B} \circ i_{D}^{B}\right)$ is a left $D$-(co)module and the quadruple $\left(r_{B_{D}}, r_{B_{D}}^{\prime}, s_{B_{D}}, s_{B_{D}}^{\prime}\right)$, where

$$
\begin{aligned}
& r_{B_{D}}:=\left(g \otimes p_{D}^{B}\right) \circ t_{B, B} \circ\left(i_{D}^{B} \otimes f\right), \quad r_{B_{D}}^{\prime}:=\left(p_{D}^{B} \otimes g\right) \circ t_{B, B}^{\prime} \circ\left(f \otimes i_{D}^{B}\right), \\
& s_{B_{D}}:=\left(p_{D}^{B} \otimes g\right) \circ t_{B, B} \circ\left(f \otimes i_{D}^{B}\right), \quad s_{B_{D}}^{\prime}:=\left(g \otimes p_{D}^{B}\right) \circ t_{B, B}^{\prime} \circ\left(i_{D}^{B} \otimes f\right),
\end{aligned}
$$

is a $\left(B_{D}, D\right)$-WO compatible with the (co)module structure in such a way that $\left(B_{D}, \varphi_{B_{D}}, \rho_{B_{D}}\right)$ is an object in ${ }_{D}^{D} \mathcal{Y D}$ ([[3] Proposition 1.19]). Since this is the case, by virtue of Theorems 3.11 and 3.12 it follows that: 
(i) $\left(B_{D}, \psi_{B_{D}}^{\lambda}, \rho_{B_{D}}^{\lambda^{-1}}\right) \in \mathcal{Y} \mathcal{D}_{D}^{D}$,

(ii) $\left(B_{D}, \psi_{B_{D}}^{\lambda^{-1}}, \rho_{B_{D}}^{\lambda}\right) \in \mathcal{Y} \mathcal{D}_{D}^{D}$,

(iii) $\left(B_{D}, \psi_{B_{D}}, \rho_{B_{D}}^{\prime}\right) \in \mathcal{Y} \mathcal{D}_{D^{\text {coop }}}^{D^{\text {coop }} \text {, }}$,

(iv) $\left(B_{D}, \psi_{B_{D}}^{\prime}, \rho_{B_{D}}\right) \in \mathcal{Y} \mathcal{D}_{D^{\text {op }}}^{D^{\text {ooop }} \text {, }}$,

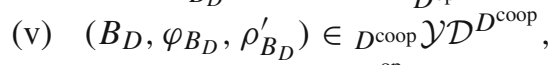

(vi) $\left(B_{D}, \psi_{B_{D}}^{\prime}, \varrho_{B_{D}}\right) \in D^{\text {op }} \mathcal{Y} \mathcal{D}_{D^{\text {op }}}$,

(vii) $\left(B_{D}, \varphi_{B_{D}}, \rho_{B_{D}}^{\lambda^{-1}}\right) \in D \mathcal{Y D}^{D}$,

(viii) $\left(B_{D}, \psi_{B_{D}}^{\lambda^{-1}}, \varrho_{B_{D}}\right) \in D \mathcal{Y} \mathcal{D}_{D}$,

where

$$
\begin{aligned}
& \psi_{B_{D}}^{\lambda}=p_{D}^{B} \circ \mu_{B} \circ t_{B, B} \circ\left(i_{D}^{B} \otimes\left(f \circ \lambda_{D}\right)\right), \quad \rho_{B_{D}}^{\lambda^{-1}}=\left(p_{D}^{B} \otimes\left(\lambda_{D}^{-1} \circ g\right)\right) \circ t_{B, B}^{\prime} \circ \delta_{B} \circ i_{D}^{B}, \\
& \psi_{B_{D}}^{\lambda^{-1}}:=p_{D}^{B} \circ \mu_{B} \circ t_{B, B}^{\prime} \circ\left(i_{D}^{B} \otimes\left(f \circ \lambda_{D}^{-1}\right)\right), \quad \rho_{B_{D}}^{\lambda}:=\left(p_{D}^{B} \otimes\left(\lambda_{D} \circ g\right)\right) \circ t_{B, B} \circ \delta_{B} \circ i_{D}^{B}, \\
& \psi_{B_{D}}:=p_{D}^{B} \circ \mu_{B} \circ t_{B, B} \circ\left(i_{D}^{B} \otimes f\right), \quad \rho_{B_{D}}^{\prime}:=\left(p_{D}^{B} \otimes g\right) \circ t_{B, B}^{\prime} \circ \delta_{B} \circ i_{D}^{B}, \\
& \psi_{B_{D}}^{\prime}:=p_{D}^{B} \circ \mu_{B} \circ t_{B, B}^{\prime} \circ\left(i_{D}^{B} \otimes f\right), \quad \rho_{B_{D}}:=\left(p_{D}^{B} \otimes g\right) \circ t_{B, B} \circ \delta_{B} \circ i_{D}^{B} .
\end{aligned}
$$

Acknowledgments The authors were supported by Ministerio de Educación (Project: MTM2010-15634) and FEDER.

Open Access This article is distributed under the terms of the Creative Commons Attribution License which permits any use, distribution, and reproduction in any medium, provided the original author(s) and the source are credited.

\section{References}

1. Alonso Álvarez, J.N.; Fernández Vilaboa, J.M.; González Rodríguez, R.: Weak Hopf algebras and weak Yang-Baxter operators. J. Algebra 320, 2101-2143 (2008)

2. Alonso Álvarez, J.N.; Fernández Vilaboa, J.M.; González Rodríguez, R.: Weak braided Hopf algebras. Indiana Univ. Math. J. 57(5), (2008)

3. Alonso Álvarez, J.N.; Fernández Vilaboa, J.M.; González Rodríguez, R.; Soneira Calvo, C.: Projections of weak braided Hopf algebras. Sci. China Math. 54(5), 877-906 (2011)

4. Alonso Álvarez, J.N.; Fernández Vilaboa, J.M.; González Rodríguez, R.; Soneira Calvo, C.: The monoidal category of Yetter-Drinfeld modules over a weak braided Hopf algebra. arXiv:1203.2474

5. Böhm, G.; Nill, F.; Szlachányi, K.: Weak Hopf algebras, I. Integral theory and $C^{*}$-structure. J. Algebra 221, $385-438$ (1999)

6. Caenepeel, S.; Wang, D.; Yin, Y.: Yetter-Drinfeld modules over weak Hopf algebras and the center construction. Ann. Univ. Ferrara - Sez. VII - Sc. Mat. 51, 69-98 (2005)

7. Joyal, A.; Street, R.: Braided tensor categories. Adv. Math. 102, 20-78 (1993)

8. Karoubi, M.: K-théorie, Les Presses de l’Université de Montréal, Montréal (1971)

9. Lambe, L.A.; Radford, D.E.: Algebraic aspects of the quantum Yang-Baxter equation. J. Algebra 154, 228-288 (1992)

10. Maclane, S.: Categories for the Working Mathematician, G.T.M, vol. 5. Springer, Berlin (1971)

11. Majid, S.: Cross products by braided groups and bosonization. J. Algebra 163, 165-190 (1994)

12. Nikshych, D.; Vainerman, A.: Finite quantum groupoids and their applications, Proc. New Directions in Hopf Algebras, Math. Sci. Res. Inst. Publ., vol. 43, Cambridge University Press, Cambridge, pp. 211-262 (2002)

13. Radford, D.E.: The structure of Hopf algebras with a projection. J. Algebra 92, 322-347 (1985)

14. Radford, D.E.: Solutions to the quantum Yang-Baxter equation and the Drinfel'd double. J. Algebra 161, 20-32 (1993)

15. Radford, D.E.; Towber, J.: Yetter-Drinfeld categories associated to an arbitrary bialgebra. J. Pure Appl. Algebra 87, 259-279 (1993)

16. Takeuchi, M.: Survey of braided Hopf algebras. Contemp. Math. 267, 301-323 (2000)

17. Yetter, D.N.: Quantum groups and representations of monoidal categories. Math. Proc. Cambridge Philos. Soc. 108, 261-290 (1990) 